\title{
Partial and Total Substitution of Dietary Fishmeal by Conventional or Genetically Modified Soybean Meal with or without Formic Acid Supplementation for Oreochromis niloticus Fingerlings
}

\author{
Ramadan Ahmed Mohamed Ali ${ }^{1}$, Ashraf Mohamed A-S Goda ${ }^{2}$, Mohamed Shaban Mohamed \\ Hassaan $^{2}$, Eman Youssef Mohammady Badiny ${ }^{2}$ \\ ${ }^{1}$ Zoology Department, Women's College for Arts, Science and Education, Ain Shams University. \\ ${ }^{2}$ Fish Nutrition Laboratory, National Institute of Oceanography and Fisheries, Fish Research Station, El- \\ Qanater El-Khayria.
}

\begin{abstract}
A feeding trial was conducted to reduce the cost of diet by using two sources of soybean meal (conventional or genetically modified) instead of the expensive commercial fishmeal (FM). In the present study, commercial FM was substituted by partial $(50 \%)$ or total $(100 \%)$ soybean. Diets were supplemented with formic acid (FA) at level of 0 or $10 \mathrm{ml} / \mathrm{kg}$ to investigate the growth performance and feed utilization of Oreochromis niloticus. The experiment was conducted with 360 Nile tilapia fingerlings (with initial weight, $22.15 \pm 2.70 \mathrm{~g}$ ). Nine isonitrogenous $25 \%$ crude protein and isocaloric $19.28 \mathrm{MJ} / \mathrm{kg}$ gross energy experimental diets were formulated. Over the 10-weeks feeding period, formic acid supplementation elevated the growth performance and feed utilization for fish fed either $50 \%$ conventional $\left(\mathrm{C}-\mathrm{SBM}_{50}\right)$ or $50 \%$ genetically modified soybean meal $\left(\mathrm{GM}_{-} \mathrm{SBM}_{50}\right)$. Serum ALT, AST, glucose and triglyceride values were lower in fish fed the control diet and conventional soybean in comparison with those fed genetically modified soybean meal. No obvious changes were found in micronucleus or in nuclear abnormalities for all diets compared with the control diet. FA supplementation improves the growth performance, feed utilization and biological abdominal parameters in fish fed either $\mathrm{C}-\mathrm{SBM}_{50}$ or $\mathrm{GM}_{-\mathrm{SBM}_{50}}$. In spite of that, genetically modified soybean elevated the levels of ALT, AST, glucose and triglyceride. These results draw our attention to the important use of organic acids in fish diets to improve the growth performance and feed utilization. As regarding the genetically modified soybean, it needs more investigation to evaluate their effect on feeding trial on fish.
\end{abstract}

Key words: Formic acid; Oreochromis niloticus; conventional soybean meal; genetically modified soybean meal.

\section{Introduction}

One of the major problems facing the fish farming industry is the increasing cost and shortage on world markets of fishmeal. The fishmeal cost represents more than $50 \%$ of the total cost of production in intensive fish culture (Adewolu and Adoti, 2010).

Corresponding Author: emy_ahmed297@yahoo.com 
The present study and the previous research works have been directed to use less expensive plant protein sources for partially or totally replacement of fishmeal (Yigit and Olmez, 2009; Abdel-Warith et al., 2013). Soybean meal is the most common plant protein used to replace fishmeal in aquaculture feeds (Yigit $\boldsymbol{e t}$ al., 2010). The soybean meal has been used due to higher protein content, well balanced amino acid profile, low price and availability (Goda $\boldsymbol{e t}$ al 2007; Hardy, 2010). Soybean meal has difficulties regarding the content of anti-nutrional factors such as trypsin, phytic acid and anti-vitamins (Kaushik and Hemre, 2008).

According to the European Community Novel Food Regulation (ECNFR), genetically modified food (GMF) is a food made from a genetic modified organism (GMO) and contains a modified genetic material. A huge variety of food crops have been genetically modified to obtain beneficial traits for herbicide tolerance, pest resistance and disease tolerance (Padgette $\boldsymbol{e t}$ al., 1995). The two most GMOs are maize and soya which represent the constituents of many foods (Meyer, 1999). The feeding studies conducted with genetic modified (GM) plants on fish were limited. They has been used in feeding of glyphosate-tolerant canola to Rainbow trout (Oncorhynchus mykiss) (Brown et al., 2003), maize to Atlantic salmon (Salmo salar) (Hemre et al., 2007) and soybean to Oreochromis niloticus (Suharman et al., 2009). Short duration of the experiments related to GM-soy has not revealed the major effects on growth performance, organ function and fish health (Hemre et al., 2005; Sagstad et al., 2008).

There are a large number of feed additives used to improve growth performance of fish like antibiotics and hormones that may cause side effects (Jouany and Morgavi, 2007). The ban for use antibiotics in livestock feed by EU since 2006 put more pressure on animal producers and poses challenge to innovate animal feed additives. Thus, a greater interest has arisen in seeking alternatives to antibiotic substances that could inhibit pathogens and act as growth promoters (Lim et al., 2010). In this context, organic acids (acidifiers) or their salts have become a promising alternative feed additive for fish feeding (Da Silva et al., 2013). Dietary formic acid enhances apparent digestibility of minerals in rainbow trout, Oncorhynchus mykiss (Vielma and Lall, 1997). Potassium diformate improved growth performance and feed conversion of hybrid tilapia (Oreochromis niloticus $\times$ O. aureus) (Zhou et al., 2009). Liebert et al. (2010) observed that adding sodium diformate to the diet of the tilapia fingerlings (Oreochromis niloticus) improved the protein efficiency ratio and protein retention. In rainbow trout (Oncorhynchus mykiss), dietary supplementation with $1 \%$ sodium diformate increased the digestibility of proteins and lipids (Morken $\boldsymbol{e t}$ al., 2011).

The present study was carried out to investigate the partial or total substitution of dietary fishmeal with conventional or genetically modified soybean meal supplemented with/without formic acid on growth, feed utilization, hematological indices and micronucleus test in $\boldsymbol{O}$. niloticus fingerlings. 


\section{Materials and methods}

\section{Fish husbandry and culture technique}

Nile tilapia fingerlings were obtained from El-Kanater El-Khayria Fish Research Station, National Institute of Oceanography and Fisheries (NIOF), Kalubiya Governorate, Egypt. A total of 360 Nile tilapia fingerlings with an average body weight $22.15 \pm 2.70 \mathrm{~g}$ were used in the present experiments. The fish were stocked into eight cement ponds $\left(0.5 \mathrm{~m}^{3}\right.$, two replicates each). Each group included 20 fish / pond. Prior to the experiment, the fish were acclimatized to the experimental conditions and fed the commercial diet $(25.48 \%$ protein) for two weeks. During the experimental period (70-days), the fish were fed on one of the nine experimental diets for 10 weeks. All fish were fed their respective diets at a level of $3 \%$ of body weight. Fish were weighted every 15 days. The daily diet was divided into three equal amounts and offered three times a day at 09:00 am, 12:00 pm and 15:00 pm.

The cement ponds were supplied with de-chlorinated tap water source. The turnover rate of water was $30 \%$ pond/day. Fish were held under natural light. Aeration was continuously provided using an air blower.

To maintain water quality at optimum range for Nile tilapia, water temperature, dissolved oxygen and $\mathrm{pH}$ were monitored during the experiment. Water temperature ranged from 27 to $28^{\circ} \mathrm{C}$, dissolved oxygen (DO) ranged between 6.32 and $6.81 \mathrm{mg} / \mathrm{l}$ and $\mathrm{pH}$ values ranged between 8.04 and 8.30 (Boyd, 1990). Water temperature was recorded daily at $13.00 \mathrm{pm}$ using a mercury thermometer suspended at 30-cm depth. Dissolved oxygen (DO) was measured at $05.00 \mathrm{pm}$ using YSI model 56 oxygen meter (YSI Company, Yellow Springs Instrument, Yellow Springs, Ohio, USA) and $\mathrm{pH}$ was measured at 09.00 am by using a $\mathrm{pH}$ meter (Orion $\mathrm{pH}$ meter, Abilene, Texas, USA).

\section{Experimental diets}

\section{Sources of diets used in the study}

Conventional soybean meal (Giza 101) (C-SBM) was supplied from Research Institute of Oil Crops, Agricultural Research Center Cairo, Egypt. Genetically modified soybean meal (GM-SBM, Glyphosate-Tolerant Soybean, GTS) was supplied by the Regional Center for Food and Feed, Ministry of Agriculture, Cairo, Egypt. Other diet components such as Fish meal, corn gluten meal, wheat bran and yellow corn were purchased from local market.

\section{Formic acid used in the study}

Formic acid $\left(\mathrm{CH}_{2} \mathrm{O}_{2}\right)$ 85\%, with molecular weight 46.03 was purchased from ElGomhouria Company for trading medicines, chemical and medical appliances, Cairo, Egypt.

\section{Formulation of experimental diets}

Nine isonitrogenous $25 \%$ crude protein and isocaloric $19.28 \mathrm{MJ} \mathrm{kg}^{-1}$ gross energy (GE) diets were formulated. The control diet had no genetically modified soybean meal (GM-SBM). In the other diets (2-9) dietary fishmeal was replaced by $50 \%$ and $100 \%$ conventional soybean 
meal (C-SBM) or GM-SBM and supplemented with formic acid at level of 0 or $10 \mathrm{ml} / \mathrm{kg}$ diet. This level of formic acid was used previously by Vielma and Lall (1997) and Sarker et al. (2012). The experimental diets are presented in table (1).

The diets were processed by blending the dry ingredients into a homogenous mixture. Pellets of $2 \mathrm{~mm}$ were made in laboratory pellet mill (CPM, California Pellet Mill Co., San Francisco, California, USA). The pelleting temperature did not exceed $40^{\circ} \mathrm{C}$ and all diets were air dried for $4 \mathrm{~h}$ (moisture content of about $10 \%$ ). All diets was packed in cellophane bags and cooled at $-4^{\circ} \mathrm{C}$ until use.

\section{Growth indices and nutrient utilization}

The following measurements and equations were carried out on fish of the present study to indicate the growth performance and biological indices of fish.

Mean final body weight (FBW) of each experimental treatment was determined by dividing total fish weight in each pond by number of fish.

Weight gain $(\mathrm{WG})=$ Final body weight $(\mathrm{g})$ - Initial body weight $(\mathrm{g})$

Specific growth rate $(\mathrm{SGR})=(\ln \mathrm{FBW}-\ln \mathrm{IBW}) / \mathrm{t} \times 100$

Where: FBW is final body weight $(\mathrm{g})$; IBW is initial body weight $(\mathrm{g})$; $\ln =$ natural logarithmic; $\mathrm{t}=$ time in days

Condition factor $(\mathrm{K})=$ weight $(\mathrm{W}) /$ length $\left(\mathrm{L}^{3}\right)$

Feed conversion ratio $($ FCR $)=$ Feed intake $(\mathrm{g}) /$ weight gain $(\mathrm{g})$

Protein efficiency ratio $(\mathrm{PER})=$ weight gain $(\mathrm{g}) /$ protein intake $(\mathrm{g})$

Protein productive value $(\mathrm{PPV})=($ protein gain $(\mathrm{g}) /$ protein intake $(\mathrm{g})) \times 100$

Fat retention $(\mathrm{FR})=($ fat gain $(\mathrm{g}) /$ fat intake $(\mathrm{g})) \times 100$

Energy retention $(\mathrm{ER})=($ energy gain $(\mathrm{kJ}) /$ energy intake $(\mathrm{kJ})) \times 100$

Hepatosomatic index (HSI \%) $=100 \times$ (liver weight $/$ whole body weight)

Spleen index $(\%)=100 \times$ (spleen weight $/$ whole body weight $)$.

\section{Analytical methods}

The final whole body proximate composition was determined from 5 fish randomly selected from each pond at the beginning and at the end of the experiment. All samples were oven-dried at $70^{\circ} \mathrm{C}$ for 3 days or at $105^{\circ} \mathrm{C}$ for 24 hours. The dried fish were ground by mixer grinder (HL1616/D Philips, India limited, $500 \mathrm{~W}$ ) and stored at $-20^{\circ} \mathrm{C}$ for subsequent analysis. The chemical composition of fish and diets samples was determined according to procedures of AOAC (1995). Samples of fish or diets were converted to ash by incineration at $550^{\circ} \mathrm{C}$ for $12 \mathrm{~h}$. Crude protein was determined by micro-Kjeldhal method, nitrogen (N) \% × 6.25 (using Kjeltech autoanalyzer, Model 1030, Tecator, Höganäs, Sweden) and crude fat by soxhlet extraction with diethyl ether $\left(400-600^{\circ} \mathrm{C}\right)$. 


\section{Analysis of blood samples}

The blood analysis was carried out in El-Amal laboratory at Kalubiya Governorate, Egypt. At the end of the experiment blood samples were collected from 4-6 fish of all treatments. The blood sample was collected with anticoagulant (10\% ethylenediaminetetraacetate, EDTA) to determine hematocrit $(\mathrm{Htc})$, hemoglobin $(\mathrm{Hb})$ and total count of white blood cells (WBCs). Another blood samples were allowed to clot and centrifuged at $3000 \mathrm{rpm}$ for 10 minutes. The serum was collected and stored at $-20^{\circ} \mathrm{C}$ until further investigation. Levels of serum aspartate aminotransferase (AST) and alanine aminotransferase (ALT) were determined according to the method described by Reitman and Frankel (1957). Total serum protein and albumin were determined according to Henry (1964) and Wotton and Freeman (1982), respectively. The total serum globulin was calculated by subtracting the total serum albumin from total serum protein according to Coles (1974). Serum glucose was determined according to Bergmeyer (1986). Serum triglyceride was determined by kits (Technicon method number SM4-0173H88).

\section{Micronucleus and nuclear abnormality analysis}

The blood smears were prepared on clean dry slides. They were air-dried overnight at room temperature and then fixed in methanol for $10 \mathrm{~min}$. and stained with 5\% Giemsa. Cells with micronuclei were scored among 2000 cells per fish under 1000X magnifications using optika microscope B-100, Italy.

The erythrocytes of $\boldsymbol{O}$. niloticus are rounded with a centrally located rounded nucleus. According to De Flora et al. (1993), the micronucleus is dark round object in cytoplasm and has the same staining intensity as the proper nucleus and separated from it. Morphological nuclear abnormalities in shape were curved, binucleated, budding or elongated and considered as a sign for cytotoxicity (Carrasco et al., 1990).

\section{Statistical analysis}

Data were statistically analyzed by ANOVA using SAS ANOVA procedure (SAS, Version 6.03, Soft Inc., Tusla, OK, USA, Statistical Analysis System, 1993). The data were submitted to one way classification variance analysis. Duncan's multiple range test was used to compare differences between treatment means when significant F-values were observed (Duncan, 1955), at $(P<0.05)$ level. All percentage data were arc-sin transformed prior to analysis (Zar, 1984), however data are presented untransformed to facilitate comparisons. 
Table (1): Composition and proximate analysis of the experimental diets

\begin{tabular}{|c|c|c|c|c|c|c|c|c|c|}
\hline \multicolumn{10}{|c|}{ Experimental diets } \\
\hline \multicolumn{2}{|l|}{ Ingredient (\%) } & \multicolumn{4}{|c|}{$\begin{array}{l}\text { Conventional soybean meal } \\
\text { (C-SBM) }\end{array}$} & \multicolumn{4}{|c|}{$\begin{array}{l}\text { Genetically modified soybean meal } \\
\text { (GM-SBM) }\end{array}$} \\
\hline \multirow{2}{*}{$\begin{array}{l}\text { Dietary SBM level } \\
\% \\
\text { Dietary formic } \\
\text { level } \mathrm{ml} / \mathrm{kg}\end{array}$} & \multirow{2}{*}{$\begin{array}{l}\text { Control } \\
\text { diet }\end{array}$} & \multicolumn{2}{|c|}{$\mathrm{C}-\mathrm{SBM}_{50}$} & \multicolumn{2}{|c|}{ C-SBM 100} & \multicolumn{2}{|c|}{$\mathrm{GM}_{-\mathrm{SBM}_{50}}$} & \multicolumn{2}{|c|}{ GM-SBM 100} \\
\hline & & 0 & 10 & 0 & 10 & 0 & 10 & 0 & 10 \\
\hline Fishmeal (60\%) & 12.0 & 6.0 & 6.0 & - & - & 6.0 & 6.0 & - & - \\
\hline C-SBM $(44 \%)$ & 24.0 & 32.2 & 32.2 & 40.4 & 40.4 & - & - & - & - \\
\hline GM-SBM $(43.5 \%)$ & - & - & - & - & - & 32.2 & 32.2 & 40.5 & 40.5 \\
\hline Corn gluten & 4.0 & 4.0 & 4.0 & 4.0 & 4.0 & 4.0 & 4.0 & 4.0 & 4.0 \\
\hline Yellow corn & 39.0 & 39.0 & 39.0 & 39.0 & 39.0 & 39.0 & 39.0 & 39.0 & 39.0 \\
\hline Wheat bran & 15.5 & 13.3 & 13.3 & 10.1 & 10.1 & 13.3 & 13.3 & 11 & 11 \\
\hline Soya oil & 3.0 & 3.0 & 3.0 & 4.0 & 4.0 & 3.0 & 3.0 & 3.0 & 3.0 \\
\hline Vit. \& $\operatorname{Min}^{1}$ & 1.5 & 1.5 & 1.5 & 1.5 & 1.5 & 1.5 & 1.5 & 1.5 & 1.5 \\
\hline Di (Ca-P) & 0.8 & 0.8 & 0.8 & 0.8 & 0.8 & 0.8 & 0.8 & 0.8 & 0.8 \\
\hline Lysine & 0.1 & 0.1 & 0.1 & 0.1 & 0.1 & 0.1 & 0.1 & 0.1 & 0.1 \\
\hline Methionine & 0.1 & 0.1 & 0.1 & 0.1 & 0.1 & 0.1 & 0.1 & 0.1 & 0.1 \\
\hline Formic acid & - & - & 10.0 & - & 10.0 & - & 10.0 & - & 10.0 \\
\hline \multicolumn{10}{|c|}{ Proximate chemical composition $(\%)$} \\
\hline Dry matter & 84.7 & 84 & 84 & 84.5 & 84.5 & 83.8 & 83.8 & 83.8 & 83.8 \\
\hline Crude protein & 25.48 & 25.27 & 25.27 & 24.96 & 24.96 & 25.38 & 25.38 & 25.11 & 25.11 \\
\hline Ether extract & 6.3 & 5.6 & 5.6 & 5.8 & 5.8 & 5.7 & 5.7 & 6.0 & 6.0 \\
\hline Crude fiber & 4.6 & 4.6 & 4.6 & 4.6 & 4.6 & 4.5 & 4.5 & 4.0 & 4.0 \\
\hline $\mathrm{NFE}^{3}$ & 62.52 & 64.13 & 64.13 & 65.04 & 65.04 & 64.02 & 64.02 & 64.69 & 64.71 \\
\hline Ash & 5.7 & 5.0 & 5.0 & 4.2 & 4.2 & 4.9 & 4.9 & 4.2 & 4.18 \\
\hline GE(MJ kg ${ }^{-1}$ diet) & 19.23 & 19.18 & 19.18 & 19.34 & 19.34 & 19.23 & 19.23 & 19.40 & 19.40 \\
\hline \multicolumn{10}{|c|}{$\begin{array}{l}{ }^{1} \text { Vitamin and mineral mix (per kg of diet): } \mathrm{MnSO}_{4}, 40 \mathrm{mg} ; \mathrm{MgO}, 10 \mathrm{mg} ; \mathrm{K}_{2} \mathrm{SO}_{4}, 40 \mathrm{mg} ; \mathrm{ZnCO}_{3}, 60 \mathrm{mg} ; \mathrm{KI}, 0.4 \mathrm{mg} \text {; } \\
\mathrm{CuSO}_{4}, 12 \mathrm{mg} \text {; Ferric citrate, } 250 \mathrm{mg} ; \mathrm{Na}_{2} \mathrm{SeO}_{3}, 0.24 \mathrm{mg} \text {; } \mathrm{Co}, 0.2 \mathrm{mg} \text {; retinol, } 40000 \mathrm{IU} \text {; cholecalciferol, } 4000 \mathrm{IU} ; \alpha- \\
\text { tocopherolacetate, } 400 \mathrm{mg} \text {; menadione, } 12 \mathrm{mg} \text {; thiamine, } 30 \mathrm{mg} \text {; riboflavin, } 40 \mathrm{mg} \text {; pyridoxine, } 30 \mathrm{mg} \text {; cyanocobalamin, } \\
80 \mathrm{mcg} \text {; ;nicotinic acid, } 300 \mathrm{mg} \text {; folic acid, } 10 \mathrm{mg} \text {; biotin, } 3 \mathrm{mg} \text {; pantothenic acid, } 100 \mathrm{mg} \text {; inositol, } 500 \mathrm{mg} \text {; ascorbic acid, } \\
500 \mathrm{mg},{ }^{2} \text { Calcium-P }=\text { Calcium propionate }(\mathrm{MW}=186.22) .{ }^{3} \mathrm{NFE} \text { (nitrogen free extract) }=100-\left(\mathrm{CP}+\mathrm{EE}+\text { Ash), }{ }^{4} \mathrm{Calculated}\right. \\
\text { using gross calorific values of } 23.63,39.52 \text { and } 17.15 \mathrm{Kj} \mathrm{g}{ }^{-1} \text { for protein, fat and carbohydrate, respectively according to } \\
\text { Brett (1973). }\end{array}$} \\
\hline
\end{tabular}

\section{Results and Discussion}

Results

In the present study, the survival rate of Nile tilapia after 10 -weeks was $100 \%$. Nile tilapia fingerlings were fed on either diets composed of control diet, 50 and $100 \%$ conventional or genetically modified soybean supplemented with / without formic acid (FA). The body weight was increased steadily with the time in fish fed different diets (Fig. 1 and 2). 
Table (2) shows the growth performance and feed utilization of Nile tilapia fed different experimental diets. FBW, FBL, K, WG and SGR were higher for fish fed either control diet or $50 \%$ conventional $\left(\mathrm{C}_{10^{-}}-\mathrm{SBM}_{50}\right)$ and genetically modified soybean meal $\left(\mathrm{GM}_{10}-\mathrm{SBM}_{50}\right)$ diets supplemented with formic acid at a levels of $10 \mathrm{ml} / \mathrm{kg}$ diet. SGR and WG increased in diets supplemented with formic acid $(10 \mathrm{ml} / \mathrm{kg}$ diet $)$ than those without formic acid supplementation (Fig. 3 and 4). Generally, the same trend was observed for protein productive value (PPV), fat retention (FR) and energy retention (ER). While, FCR decreased with formic acid supplementation. The lowest growth performance and feed utilization was recorded in diets containing $100 \%$ un-supplemented conventional soybean $\left(\mathrm{C}_{0}-\mathrm{SBM}_{100}\right)$ and $50 \%$ genetically modified soybean meal un-supplemented with FA $\left(\mathrm{GM}_{0}-\mathrm{SBM}_{50}\right)$.

Biological abdominal parameters revealed in table (3) that the highest hepatosomatic index (HSI) and liver weight were recorded for fish fed the control diet followed by the diet containing $\mathrm{C}_{0}-\mathrm{SBM}_{100}$. While, the lowest values were observed for fish fed diet containing $\mathrm{GM}_{0^{-}}$ $\mathrm{SBM}_{50}$. Fish fed the diet containing $100 \%$ either $\mathrm{C}_{0}-\mathrm{SBM}$ or $\mathrm{GM}_{0}-\mathrm{SBM}$ without formic acid supplementation recorded significantly $(P<0.05)$ higher spleen weight and spleen index. The lowest values were observed in fish fed the diet containing $100 \%$ genetically modified soybean $\left(\mathrm{GM}_{10}-\mathrm{SBM}_{100}\right)$ supplemented with $10 \mathrm{ml} / \mathrm{kg}$ formic acid. Intestine length of $100 \%$ conventional soybean $\left(\mathrm{C}_{0}-\mathrm{SBM}_{100}\right)$ un-supplemented with formic acid and $100 \%$ genetically modified soybean $\left(\mathrm{GM}_{0}-\mathrm{SBM}_{100}\right)$ had the maximum intestine length (Fig. 5).

Hematological and biochemical blood analysis are presented in table (4). Hemoglobin $\mathrm{Hb}$ ) and hematocrit (Hct) of Nile tilapia increased with dietary incorporation of formic acid compared to the fish fed the diet without formic acid supplementation. Generally, the mean white blood cell (WBCs) and blood neutrophil counts were decreased with formic acid supplementation than in fish fed the experimental diets. But, they increased for diet containing $100 \%$ genetically modified soybean $\left(\mathrm{GM}_{10}-\mathrm{SBM}_{100}\right)$. The opposite trend was recorded for lymphocyte count increased with formic acid supplementation for the experimental diets expect fish fed $50 \%$ genetically modified soybean $\left(\mathrm{GM}_{10}-\mathrm{SBM}_{50}\right)$ decreased with supplemented formic acid.

No clear trend was observed for the levels of total protein, albumin and globulin, in the serum of Nile tilapia table (4). Serum globulin level decreased for fish fed $100 \%$ conventional soybean $\left(\mathrm{C}-\mathrm{SBM}_{100}\right)$ and $50 \%$ genetically modified soybean $\left(\mathrm{GM}-\mathrm{SBM}_{50}\right)$ diet with adding formic acid compared to other experimental diets.

The values of total serum triglyceride, glucose, ALT and AST increased in fish fed either conventional (C-SBM) or genetically modified soybean meal (GM-SBM) with or without formic acid supplementation compared to control diet (Table 4 and Fig. 6, 7, 8 and 9). 
The data of micronucleus and nuclear abnormalities in erythrocytes of Nile tilapia diets are shown in table (5). Micronuclei are small according to the size of the containing cell (Fig. 10). No differences were observed in micronuclei averages among fish fed all experimental diets. Any shapes in nucleus other than the round shape was considered as nuclear abnormalities. In addition, the abnormal forms appeared as curved, binucleated, budding and elongated (Fig. 11). The nuclear abnormalities ranged from 2 to 4 cells / 2000 erythrocytes.

The whole body proximate composition of Nile tilapia fed different experimental diets is shown in table (6). Nile tilapia fed diet containing $\mathrm{GM}_{0}-\mathrm{SBM}_{100}$ and $\mathrm{C}_{10}-\mathrm{SBM}_{100}$ supplemented with formic acid showed the highest significant $(P<0.05)$ values of body moisture content. Fish fed $\mathrm{C}_{10}-\mathrm{SBM}_{100}$ diet supplemented with $10 \mathrm{ml} / \mathrm{kg}$ formic acid recorded the highest significant $(P$ $<0.05)$ ash percentage. The whole body lipid content $(\%)$ was recorded the highest value for fish fed $\mathrm{C}_{10}-\mathrm{SBM}_{50}$ diet supplemented with $10 \mathrm{ml} / \mathrm{kg}$ formic acid. The protein content value was highest for fish fed $\mathrm{GM}_{10}-\mathrm{SBM}_{100}$ diet supplemented with $10 \mathrm{ml} / \mathrm{kg}$ formic acid. 
Table (2): Growth performance and feed utilization of $O$. niloticus fed control diet or replaced by $50 \%$ or $100 \%$ of C-SBM or GM-SBM. The soybean diets supplemented with formic acid at 0 or $10 \mathrm{ml} / \mathrm{kg} \mathrm{diet}$.

\begin{tabular}{|c|c|c|c|c|c|c|c|c|c|c|}
\hline \multicolumn{11}{|c|}{ Experimental diets } \\
\hline \multirow{3}{*}{$\begin{array}{l}\text { Ingredients }(\%) \\
\text { Dietary SBM level \% } \\
\text { Dietary formic acid level ml/ } \\
\text { kg diet }\end{array}$} & \multirow{3}{*}{$\begin{array}{l}\text { Control } \\
\text { diet }\end{array}$} & \multicolumn{4}{|c|}{$\begin{array}{l}\text { Conventional soybean meal } \\
\text { (C-SBM) }\end{array}$} & \multicolumn{4}{|c|}{$\begin{array}{l}\text { Genetically modified soybean meal } \\
\text { (GM-SBM) }\end{array}$} & \multirow{3}{*}{$\pm \mathrm{SE}$} \\
\hline & & \multicolumn{2}{|c|}{ C-SBM 50} & \multicolumn{2}{|c|}{${\mathrm{C}-\mathrm{SBM}_{100}}_{1}$} & \multicolumn{2}{|c|}{$\mathrm{GM}^{-\mathrm{SBM}_{50}}$} & \multicolumn{2}{|c|}{$\mathrm{GM}^{-\mathrm{SBM}_{100}}$} & \\
\hline & & 0 & 10 & 0 & 10 & 0 & 10 & 0 & 10 & \\
\hline Initial body weight $(\mathrm{g})$ & & $22.14^{\mathrm{a}}$ & $22.14^{\mathrm{a}}$ & $22.15^{\mathrm{a}}$ & $22.16^{\mathrm{a}}$ & $22.17^{\mathrm{a}}$ & $22.13^{\mathrm{a}}$ & $22.11^{\mathrm{a}}$ & $22.18^{\mathrm{a}}$ & 0.43 \\
\hline Final body weight (g) & $52.53^{\mathrm{a}}$ & $49.01^{\mathrm{b}}$ & $50.66^{\mathrm{ab}}$ & $44.12^{\mathrm{c}}$ & $45.09^{\mathrm{c}}$ & $43.91^{\mathrm{d}}$ & $50.72^{\mathrm{ab}}$ & $44.67^{\mathrm{c}}$ & $44.74^{\mathrm{c}}$ & 0.76 \\
\hline Final body length $(\mathrm{cm})$ & $14.13^{\mathrm{a}}$ & $12.87^{\mathrm{bc}}$ & $13.15^{\mathrm{bc}}$ & $12.95^{\mathrm{bc}}$ & $12.79^{\mathrm{c}}$ & $12.85^{\mathrm{bc}}$ & $13.32^{\mathrm{b}}$ & $13.05^{\mathrm{bc}}$ & $13.23^{\mathrm{bc}}$ & 0.16 \\
\hline Condition factor & $1.33^{\mathrm{a}}$ & $1.28^{\mathrm{b}}$ & $1.31^{\mathrm{ab}}$ & $1.14^{\mathrm{cd}}$ & $1.18^{\mathrm{c}}$ & $1.13^{\mathrm{cd}}$ & $1.28^{\mathrm{ab}}$ & $1.14^{\mathrm{cd}}$ & $1.12^{\mathrm{d}}$ & 0.02 \\
\hline Specific growth $\mathrm{r}$ & $1.20^{\mathrm{a}}$ & $1.11^{\mathrm{a}}$ & $1.15^{\mathrm{a}}$ & $0.96^{\mathrm{b}}$ & $0.99^{\mathrm{b}}$ & $0.95^{\mathrm{b}}$ & $1.16^{\mathrm{a}}$ & $0.98^{\mathrm{b}}$ & $0.98^{\mathrm{b}}$ & 0.03 \\
\hline Weight gain $(\mathrm{g})$ & $30.31^{\mathrm{a}}$ & $26.87^{\mathrm{b}}$ & $28.53^{\mathrm{ab}}$ & $21.98^{\mathrm{c}}$ & $22.93^{\mathrm{c}}$ & $21.74^{\mathrm{c}}$ & $28.60^{\mathrm{ab}}$ & $22.56^{\mathrm{c}}$ & $22.57^{\mathrm{c}}$ & 0.98 \\
\hline Feed intake (g/fish) & $55.30^{\mathrm{a}}$ & $50.73^{\mathrm{bc}}$ & $53.78^{\mathrm{ab}}$ & $49.23^{\mathrm{c}}$ & $47.26^{\mathrm{c}}$ & $53.32^{\mathrm{ab}}$ & $53.08^{\mathrm{ab}}$ & $53.28^{\mathrm{ab}}$ & $53.93^{\mathrm{ab}}$ & 1.08 \\
\hline Feed conversion ratio & $1.83^{\mathrm{c}}$ & $1.89^{\mathrm{c}}$ & $1.89^{\mathrm{c}}$ & $2.25^{\mathrm{ab}}$ & $2.07^{\mathrm{bc}}$ & $2.46^{\mathrm{a}}$ & $1.86^{\mathrm{c}}$ & $2.36^{\mathrm{a}}$ & $2.39^{\mathrm{a}}$ & 0.07 \\
\hline Protein efficiency ratio & $2.19^{\mathrm{a}}$ & $2.12^{\mathrm{ab}}$ & $2.12^{\mathrm{ab}}$ & $1.79^{\mathrm{cd}}$ & $1.94^{\mathrm{bc}}$ & $1.63^{\mathrm{d}}$ & $2.16^{\mathrm{ab}}$ & $1.70^{\mathrm{d}}$ & $1.68^{\mathrm{d}}$ & 0.07 \\
\hline Protein productive value $(\%)$ & $31.56^{\mathrm{ab}}$ & $31.42^{\mathrm{ab}}$ & $29.22^{\mathrm{b}}$ & $23.92^{\mathrm{c}}$ & $26.00^{\mathrm{c}}$ & $23.58^{\mathrm{c}}$ & $33.09^{\mathrm{a}}$ & $23.98^{\mathrm{c}}$ & $25.38^{\mathrm{c}}$ & 0.94 \\
\hline Fat retention $(\%)$ & $51.11^{\mathrm{a}}$ & $50.72^{\mathrm{a}}$ & $42.04^{\mathrm{bc}}$ & $33.13^{\mathrm{de}}$ & $36.77^{\mathrm{cd}}$ & $35.93^{\mathrm{cd}}$ & $45.00^{\mathrm{b}}$ & $25.51^{\mathrm{f}}$ & $28.70^{\text {ef }}$ & 1.99 \\
\hline Energy retention (\%) & $16.52^{\mathrm{a}}$ & $16.37^{\mathrm{a}}$ & $14.21^{\mathrm{b}}$ & $11.20^{\mathrm{cd}}$ & $12.87^{\mathrm{bc}}$ & $11.46^{\mathrm{cd}}$ & $16.24^{\mathrm{a}}$ & $10.82^{\mathrm{d}}$ & $11.33^{\mathrm{cd}}$ & 0.57 \\
\hline
\end{tabular}

Data are presented as means \pm standard error (SE) of duplicates.

Means in the same rows with the same letter are not significantly different $(\mathrm{P}>0.05)$. 
Table (3): Biological abdominal parameters of $O$. niloticus fed control diet or replaced by $50 \%$ or $100 \%$ of C-SBM or GM-SBM. The soybean diets supplemented with formic acid at 0 or $10 \mathrm{ml} / \mathrm{kg} \mathrm{diet}$.

\begin{tabular}{|c|c|c|c|c|c|c|c|c|c|c|}
\hline \multicolumn{11}{|c|}{ Experimental diets } \\
\hline \multirow{3}{*}{$\begin{array}{l}\text { Ingredients (\%) } \\
\text { Dietary SBM level \% } \\
\text { Dietary formic acid level } \\
\mathrm{ml} / \mathrm{kg} \text { diet }\end{array}$} & \multirow{3}{*}{$\begin{array}{l}\text { Control } \\
\text { diet }\end{array}$} & \multicolumn{4}{|c|}{$\begin{array}{c}\text { Conventional soybean meal } \\
(\mathrm{C}-\mathrm{SBM})\end{array}$} & \multicolumn{4}{|c|}{$\begin{array}{l}\text { Genetically modified soybean meal } \\
\text { (GM-SBM) }\end{array}$} & \multirow{3}{*}{$\pm \mathrm{SE}$} \\
\hline & & \multicolumn{2}{|c|}{ C-SBM 50} & \multicolumn{2}{|c|}{ C-SBM 100} & \multicolumn{2}{|c|}{$\mathrm{GM}^{-\mathrm{SBM}_{50}}$} & \multicolumn{2}{|c|}{$\mathrm{GM}^{-S B M} \mathrm{M}_{100}$} & \\
\hline & & 0 & 10 & 0 & 10 & 0 & 10 & 0 & 10 & \\
\hline Body biomass (g) & $53.24^{\mathrm{bc}}$ & $38.56^{\mathrm{e}}$ & $50.20^{\mathrm{cd}}$ & $61.06^{\mathrm{a}}$ & $41.33^{\mathrm{e}}$ & $37.73^{\mathrm{e}}$ & $38.48^{\mathrm{e}}$ & $57.67^{\mathrm{ab}}$ & $45.88^{\mathrm{d}}$ & 1.49 \\
\hline Liver weight (g) & $2.13^{\mathrm{a}}$ & $0.87^{\mathrm{d}}$ & $1.31^{\mathrm{bc}}$ & $2.12^{\mathrm{a}}$ & $0.75^{\mathrm{d}}$ & $0.67^{\mathrm{d}}$ & $1.51^{\mathrm{b}}$ & $1.36^{\mathrm{bc}}$ & $1.03^{\mathrm{cd}}$ & 0.12 \\
\hline Hepatosomatic index $\%$ & $4.01^{\mathrm{a}}$ & $2.26^{\mathrm{bc}}$ & $2.61^{\mathrm{b}}$ & $3.47^{\mathrm{a}}$ & $1.80^{\mathrm{c}}$ & $1.78^{\mathrm{c}}$ & $3.92^{\mathrm{a}}$ & $2.36^{\mathrm{bc}}$ & $2.24^{\mathrm{bc}}$ & 0.23 \\
\hline Spleen weight (gm) & $0.11^{\mathrm{ab}}$ & $0.11^{\mathrm{ab}}$ & $0.06^{\mathrm{d}}$ & $0.12^{\mathrm{a}}$ & $0.09^{\mathrm{bc}}$ & $0.07^{\mathrm{cd}}$ & $0.07^{\mathrm{cd}}$ & $0.12^{\mathrm{a}}$ & $0.05^{\mathrm{d}}$ & 0.01 \\
\hline Spleen index $\%$ & $0.21^{\mathrm{b}}$ & $0.29^{\mathrm{a}}$ & $0.12^{\mathrm{cd}}$ & $0.20^{\mathrm{b}}$ & $0.22^{\mathrm{b}}$ & $0.19^{\mathrm{b}}$ & $0.18^{\mathrm{cb}}$ & $0.22^{\mathrm{b}}$ & $0.11^{\mathrm{d}}$ & 0.02 \\
\hline Length of intestine $(\mathrm{cm})$ & $60.5^{\mathrm{cd}}$ & $65.5^{\mathrm{c}}$ & $56.00^{\mathrm{de}}$ & $81.1^{\mathrm{ab}}$ & $63.5^{\mathrm{c}}$ & $54.02^{\mathrm{e}}$ & $47.07^{\mathrm{f}}$ & $83.03^{\mathrm{a}}$ & $76.5^{\mathrm{b}}$ & 1.67 \\
\hline
\end{tabular}

Data are presented as means \pm standard error (SE) of duplicates.

Means in the same rows with the same letter are not significantly different $(\mathrm{P}>0.05)$. 
Table (4): Blood picture and blood chemistry of $O$. niloticus fed control diet or replaced by $50 \%$ or $100 \%$ of C-SBM or GM-SBM. The soybean diets supplemented with formic acid at 0 or $10 \mathrm{ml} / \mathrm{kg}$ diet.

\begin{tabular}{|c|c|c|c|c|c|c|c|c|c|c|}
\hline \multicolumn{11}{|c|}{ Experimental diets } \\
\hline \multirow{3}{*}{$\begin{array}{l}\text { Ingredients (\%) } \\
\text { Dietary SBM level \% } \\
\text { Dietary formic acid level } \\
\mathrm{ml} / \mathrm{kg} \text { diet }\end{array}$} & \multirow{3}{*}{$\begin{array}{c}\text { Control } \\
\text { diet }\end{array}$} & \multicolumn{4}{|c|}{$\begin{array}{l}\text { Conventional soybean meal } \\
\text { (C-SBM) }\end{array}$} & \multicolumn{4}{|c|}{$\begin{array}{l}\text { Genetically modified soybean meal } \\
\text { (GM-SBM) }\end{array}$} & \multirow{3}{*}{$\pm \mathrm{SE}$} \\
\hline & & \multicolumn{2}{|c|}{$\mathrm{C}-\mathrm{SBM}_{50}$} & \multicolumn{2}{|c|}{$\mathrm{C}-\mathrm{SBM}_{100}$} & \multicolumn{2}{|c|}{$\mathrm{GM}^{-\mathrm{SBM}_{50}}$} & \multicolumn{2}{|c|}{ GM-SBM $_{100}$} & \\
\hline & & 0 & 10 & 0 & 10 & 0 & 10 & 0 & 10 & \\
\hline Hemoglobin (g/l) & $10.92^{c}$ & $11.66^{\mathrm{ab}}$ & $11.75^{\mathrm{ab}}$ & $10.58^{\mathrm{cd}}$ & $11.38^{\mathrm{b}}$ & $11.48^{\mathrm{ab}}$ & $11.85^{\mathrm{a}}$ & $10.33^{\mathrm{d}}$ & $10.45^{\mathrm{d}}$ & 0.13 \\
\hline Haematocrite (\%) & $34.95^{\mathrm{c}}$ & $37.32^{\mathrm{ab}}$ & $37.60^{\mathrm{a}}$ & $33.86^{\mathrm{cd}}$ & $36.40^{\mathrm{b}}$ & $36.74^{\mathrm{b}}$ & $37.92^{\mathrm{a}}$ & $33.06^{\mathrm{d}}$ & $33.44^{\mathrm{d}}$ & 0.41 \\
\hline WBC $\left(10^{3}\right.$ cell $\left.\mathrm{mm}^{-3}\right)$ & $10.96^{\mathrm{ab}}$ & $10.70^{\mathrm{bc}}$ & $10.55^{\mathrm{bc}}$ & $11.56^{\mathrm{a}}$ & $11.05^{\mathrm{ab}}$ & $10.91^{\mathrm{abc}}$ & $10.60^{\mathrm{bc}}$ & $10.22^{\mathrm{c}}$ & $11.59^{\mathrm{a}}$ & 0.20 \\
\hline Lymphocyte (\%) & $57.77^{\mathrm{ab}}$ & $59.16^{\mathrm{ab}}$ & $60.45^{\mathrm{a}}$ & $57.63^{\mathrm{b}}$ & $59.18^{\mathrm{ab}}$ & $60.26^{\mathrm{ab}}$ & $59.12^{\mathrm{ab}}$ & $58.65^{\mathrm{ab}}$ & $59.13^{\mathrm{ab}}$ & 0.76 \\
\hline Neutrophil (\%) & $20.13^{\mathrm{a}}$ & $19.24^{\mathrm{ab}}$ & $18.62^{\mathrm{b}}$ & $20.20^{\mathrm{a}}$ & $19.61^{\mathrm{ab}}$ & $19.50^{\mathrm{ab}}$ & $19.35^{\mathrm{ab}}$ & $18.75^{\mathrm{b}}$ & $19.50^{\mathrm{ab}}$ & 0.36 \\
\hline Monocyte (\%) & $17.62^{\mathrm{c}}$ & $17.22^{\mathrm{c}}$ & $18.13^{\mathrm{bc}}$ & $19.07^{\mathrm{ab}}$ & $19.46^{\mathrm{a}}$ & $17.67^{\mathrm{c}}$ & $18.10^{\mathrm{bc}}$ & $19.05^{\mathrm{ab}}$ & $19.05^{\mathrm{ab}}$ & 0.36 \\
\hline Serum Protein $(\mathrm{g} / \mathrm{dl})$ & $3.30^{\mathrm{ab}}$ & $2.90^{\mathrm{b}}$ & $3.40^{\mathrm{a}}$ & $3.10^{\mathrm{ab}}$ & $2.71^{\mathrm{d}}$ & $2.90^{\mathrm{b}}$ & $2.50^{\mathrm{d}}$ & $2.55^{\mathrm{d}}$ & $3.00^{\mathrm{ab}}$ & 0.14 \\
\hline Serum Albumin (A) (g/dl) & $1.85^{\mathrm{a}}$ & $1.45^{\mathrm{c}}$ & $1.65^{\mathrm{b}}$ & $1.35^{\mathrm{c}}$ & $1.25^{\mathrm{d}}$ & $1.40^{\mathrm{c}}$ & $1.25^{\mathrm{d}}$ & $1.15^{\mathrm{d}}$ & $1.30^{\mathrm{c}}$ & 0.05 \\
\hline Serum globulin $(\mathrm{G})(\mathrm{g} / \mathrm{dl})$ & $1.45^{\mathrm{ab}}$ & $1.45^{\mathrm{ab}}$ & $1.75^{\mathrm{a}}$ & $1.75^{\mathrm{a}}$ & $1.46^{\mathrm{ab}}$ & $1.50^{\mathrm{ab}}$ & $1.25^{\mathrm{b}}$ & $1.40^{\mathrm{ab}}$ & $1.70^{\mathrm{a}}$ & 0.10 \\
\hline $\mathrm{A} / \mathrm{g}$ ratio & $1.28^{\mathrm{a}}$ & $1.00^{\mathrm{b}}$ & $0.94^{\mathrm{bc}}$ & $0.77^{\mathrm{c}}$ & $0.86^{\mathrm{bc}}$ & $0.94^{\mathrm{bc}}$ & $1.00^{\mathrm{b}}$ & $0.83^{b c}$ & $0.77^{\mathrm{c}}$ & 0.05 \\
\hline Serum triglyceride $(\mathrm{mg} / \mathrm{dl})$ & $182.50^{\mathrm{f}}$ & $191.00^{\mathrm{d}}$ & $187.00^{\mathrm{e}}$ & $205.50^{\mathrm{ab}}$ & $207.50^{\mathrm{a}}$ & $201.50^{\mathrm{c}}$ & $202.00^{c}$ & $204.00^{\mathrm{b}}$ & $204.50^{\mathrm{b}}$ & 0.97 \\
\hline Serum glucose (mg/gl) & $71.50^{\mathrm{f}}$ & $74.00^{\mathrm{e}}$ & $73.50^{\mathrm{ef}}$ & $79.50^{\mathrm{d}}$ & $81.50^{\mathrm{d}}$ & $97.50^{\mathrm{b}}$ & $94.00^{c}$ & $100.00^{\mathrm{a}}$ & $99.00^{\mathrm{ab}}$ & 0.63 \\
\hline Serum AST((U/L) & $91.00^{c}$ & $93.00^{c}$ & $92.00^{c}$ & $92.95^{\mathrm{c}}$ & $91^{\mathrm{c}}$ & $103.10^{\mathrm{b}}$ & $101.55^{\mathrm{b}}$ & $106^{\mathrm{a}}$ & $105.5^{\mathrm{a}}$ & 0.62 \\
\hline Serum ALT((U/L) & $20.05^{\mathrm{e}}$ & $22.10^{\mathrm{d}}$ & $19.67^{\mathrm{e}}$ & $22.08^{\mathrm{d}}$ & $23.58^{\mathrm{c}}$ & $27.97^{\mathrm{b}}$ & $28.77^{\mathrm{b}}$ & $30.46^{\mathrm{a}}$ & $30.81^{\mathrm{a}}$ & 0.35 \\
\hline
\end{tabular}

Data are presented as means \pm standard error (SE) of duplicates.

Means in the same rows with the same letter are not significantly different $(\mathrm{P}>0.05)$. 
Table (5): Frequency of micronuclated erythrocytes and nuclear abnormalities/ 2000 erythrocytes of $O$. niloticus fed control diet or replaced by $50 \%$ or $100 \%$ of C-SBM or GM-SBM. The soybean diets supplemented with formic acid at 0 or $10 \mathrm{ml} / \mathrm{kg}$ diet.

\begin{tabular}{|c|c|c|c|c|c|c|c|c|c|c|}
\hline \multicolumn{11}{|c|}{ Experimental diets } \\
\hline \multicolumn{3}{|l|}{ Ingredients (\%) } & \multicolumn{4}{|c|}{$\begin{array}{c}\text { Conventional soybean meal } \\
\text { (C-SBM) }\end{array}$} & \multicolumn{4}{|c|}{$\begin{array}{l}\text { Genetically modified soybean meal } \\
\text { (GM-SBM) }\end{array}$} \\
\hline \multicolumn{2}{|l|}{ Dietary SBM level \% } & \multirow[t]{2}{*}{ Control diet } & \multicolumn{2}{|c|}{$\mathrm{C}-\mathrm{SBM}_{50}$} & \multicolumn{2}{|c|}{$\mathrm{C}-\mathrm{SBM}_{100}$} & \multicolumn{2}{|c|}{$\mathrm{GM}^{-\mathrm{SBM}_{50}}$} & \multicolumn{2}{|c|}{ GM-SBM $_{100}$} \\
\hline \multicolumn{2}{|c|}{ Dietary formic acid level $\mathrm{ml} / \mathrm{kg}$ diet } & & 0 & 10 & 0 & 10 & 0 & 10 & 0 & 10 \\
\hline \multicolumn{2}{|c|}{ Micronucleus/ 2000 cells } & 2 & 1 & 2 & 0 & 1 & 1 & 0 & 1 & 0 \\
\hline \multirow{5}{*}{$\begin{array}{l}\text { Nuclear abnormalities } \\
\text { / } 2000 \text { cells }\end{array}$} & Curved & 3 & 2 & 3 & 1 & 1 & 2 & 1 & 3 & 1 \\
\hline & bionucleated & 0 & 0 & 0 & 2 & 1 & 0 & 1 & 1 & 2 \\
\hline & budding & 1 & 0 & 0 & 0 & 0 & 1 & 0 & 0 & 0 \\
\hline & elongated & 0 & 0 & 1 & 0 & 0 & 0 & 0 & 0 & 0 \\
\hline & Total & 4 & 2 & 4 & 3 & 2 & 3 & 2 & 4 & 3 \\
\hline
\end{tabular}

2000 erythrocytes were examined from 4 Nile tilapia; 500 each / diet trial. 
Table (6): Whole body proximate composition of $O$. niloticus fed control diet or replaced by $50 \%$ or $100 \%$ of C-SBM or GM-SBM. The soybean diets supplemented with formic acid at 0 or $10 \mathrm{ml} / \mathrm{kg} \mathrm{diet.}$

\begin{tabular}{|c|c|c|c|c|c|c|c|c|c|c|}
\hline \multicolumn{11}{|c|}{ Experimental diet } \\
\hline \multirow{3}{*}{$\begin{array}{l}\text { Ingredient }(\mathrm{g} / \mathrm{kg}) \\
\text { Dietary SBM level \% } \\
\text { Dietary formic acid } \\
\text { level } \mathrm{ml} / \mathrm{kg}\end{array}$} & \multirow{3}{*}{$\begin{array}{c}\text { Control } \\
\text { diet }\end{array}$} & \multicolumn{4}{|c|}{$\begin{array}{c}\text { Conventional soybean meal } \\
(\text { C- SBM })\end{array}$} & \multicolumn{4}{|c|}{$\begin{array}{l}\text { Genetically modified soybean meal } \\
\text { (GM-SBM) }\end{array}$} & \multirow{3}{*}{$\pm \mathrm{SE}$} \\
\hline & & \multicolumn{2}{|c|}{$\mathrm{C}-\mathrm{SBM}_{50}$} & \multicolumn{2}{|c|}{ C-SBM 100} & \multicolumn{2}{|c|}{$\mathrm{GM}^{-\mathrm{SBM}_{50}}$} & \multicolumn{2}{|c|}{$\mathrm{GM}^{-S_{B M}}{ }_{100}$} & \\
\hline & & 0 & 10 & 0 & 10 & 0 & 10 & 0 & 10 & \\
\hline Moisture \% & $73.40^{\mathrm{b}}$ & $73.63^{b}$ & $74.95^{\mathrm{ab}}$ & $74.64^{\mathrm{ab}}$ & $75.70^{\mathrm{a}}$ & $73.91^{\mathrm{b}}$ & $73.72^{\mathrm{b}}$ & $75.96^{\mathrm{a}}$ & $74.79^{\mathrm{ab}}$ & 0.51 \\
\hline Ash \% & $14.18^{\mathrm{def}}$ & $13.61^{\mathrm{f}}$ & $14.72^{\mathrm{de}}$ & $16.04^{\mathrm{b}}$ & $17.03^{\mathrm{a}}$ & $14.05^{\mathrm{ef}}$ & $14.89^{\mathrm{cd}}$ & $15.61^{\mathrm{bc}}$ & $15.56^{\mathrm{bc}}$ & 0.25 \\
\hline Lipid \% & $25.99^{\mathrm{a}}$ & $23.40^{\mathrm{b}}$ & $27.28^{\mathrm{a}}$ & $21.69^{\mathrm{b}}$ & $23.13^{\mathrm{b}}$ & $23.18^{\mathrm{b}}$ & $24.4^{\mathrm{ab}}$ & $20.51^{\mathrm{c}}$ & $20.19^{c}$ & 0.51 \\
\hline Protein \% & $53.74^{\mathrm{b}}$ & $55.29^{\mathrm{b}}$ & $55.42^{\mathrm{b}}$ & $53.93^{\mathrm{b}}$ & $54.37^{\mathrm{b}}$ & $55.52^{\mathrm{ab}}$ & $57.54^{\mathrm{ab}}$ & $57.92^{\mathrm{ab}}$ & $59.48^{\mathrm{a}}$ & 0.97 \\
\hline
\end{tabular}

Data are presented as means \pm standard error (SE) of duplicates.

Means in the same rows with the same letter are not significantly different $(\mathrm{P}>0.05)$. 


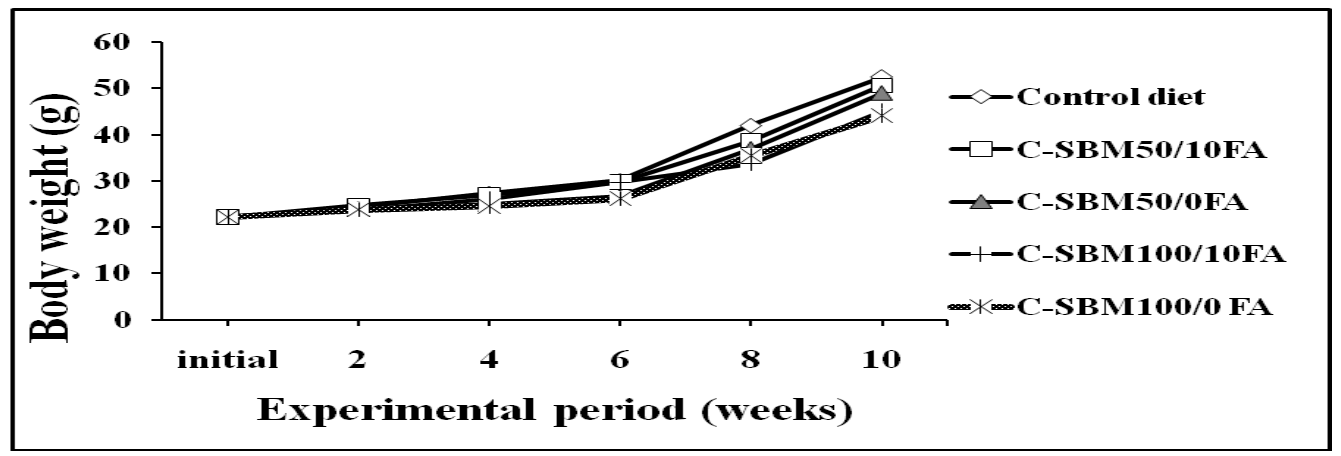

Fig.(1) Body weight of fish fed conventional soybean supplemented with / without formic acid (FA, $10 \mathrm{ml} / \mathrm{kg}$ diet). The body weight was increased steadily with the time in fish fed different diets. The highest FBW was recorded for fish fed either control diet or $50 \%$ conventional soybean $\left(\mathrm{C}-\mathrm{SBM}_{50}\right)$ diets supplemented with formic acid at a level of $10 \mathrm{ml} / \mathrm{kg}$ diet.

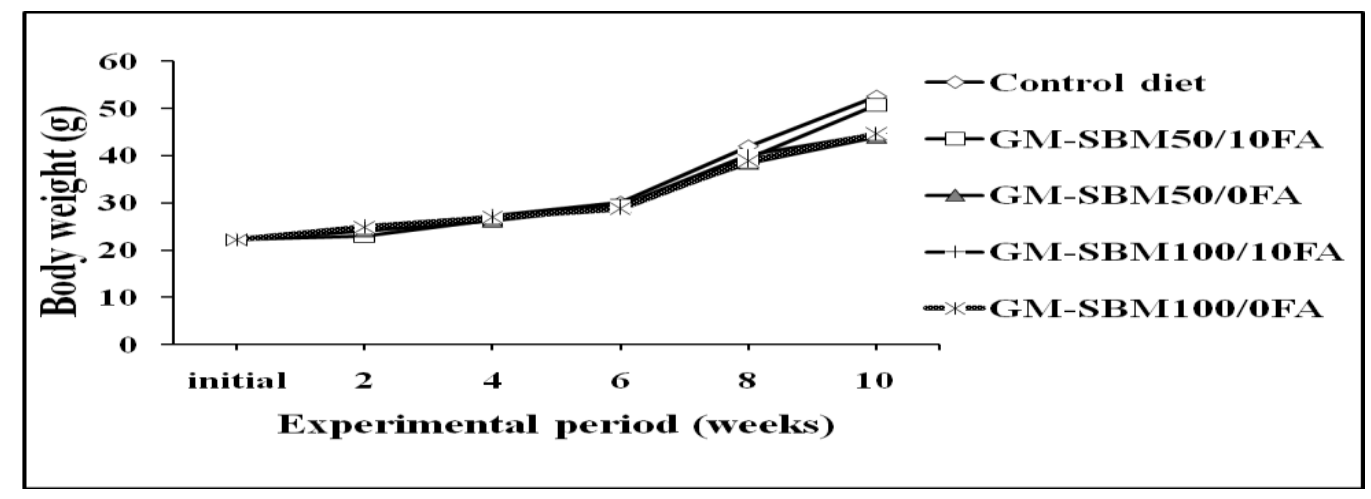

Fig. (2) Body weight of fish fed genetically modified soybean supplemented with / without formic acid supplementation $(10 \mathrm{ml} / \mathrm{kg}$ diet $)$. The body weight was increased steadily with the time in fish fed different diets. The highest FBW was recorded for fish fed either control diet or genetically modified soybean (GM-SBM 50 ) diet supplemented with formic acid at a level of $10 \mathrm{ml} / \mathrm{kg}$ diet.

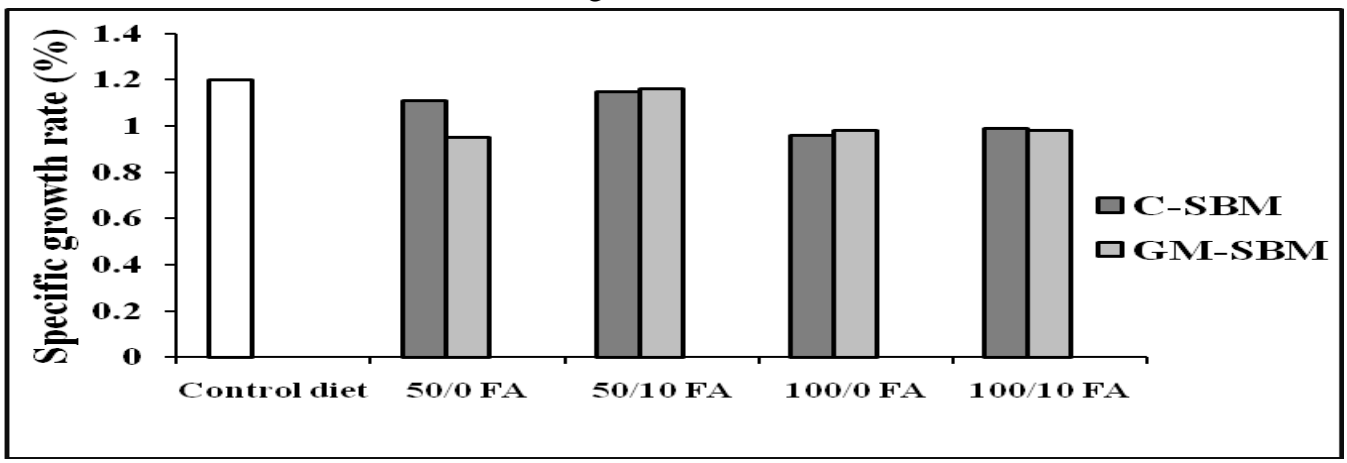

Fig. (3): Specific growth rate (\%) of Nile tilapia fed control diet and diets substituted with partial and total of dietary FM by C-SBM or GM-SBM with or without FA supplementation. Note that, SGR was relatively increased with the addition of formic for C-SBM or GM-SBM. The highest values of SGR were recorded for fish fed control diet and diets containing either 50\% C-SBM or GM-SBM supplemented with formic acid. 


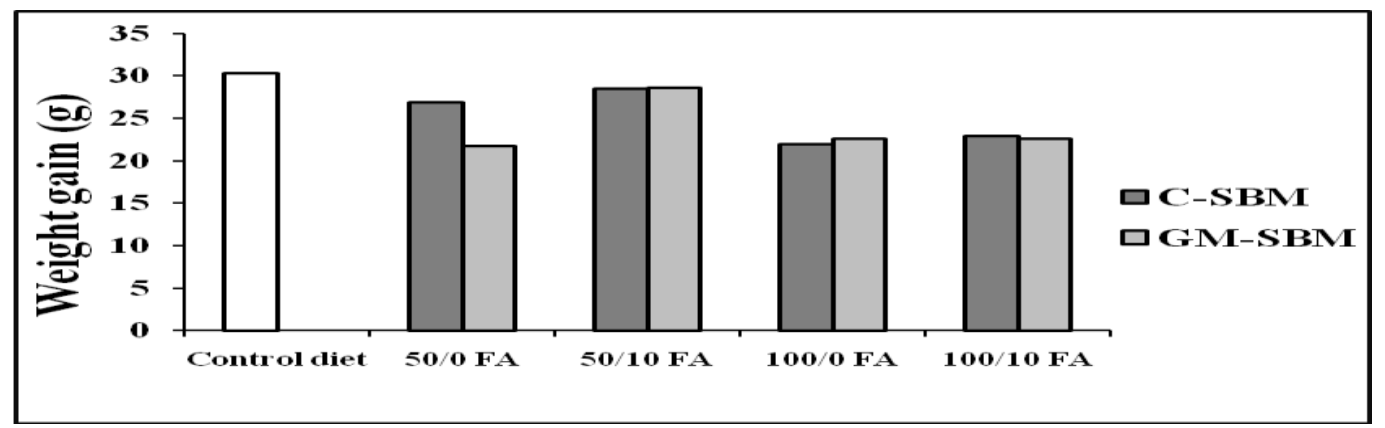

Fig. (4): Weight gain (g) of Nile tilapia fed control diet and diets substituted with partial and total of dietary FM by C-SBM or GM-SBM with or without FA supplementation. Note that, WG was relatively increased with the addition of formic acid for C-SBM or GM-SBM. The highest values of WG were recorded for fish fed control diet and diet containing either 50\% C-SBM or GM-SBM supplemented with formic acid.

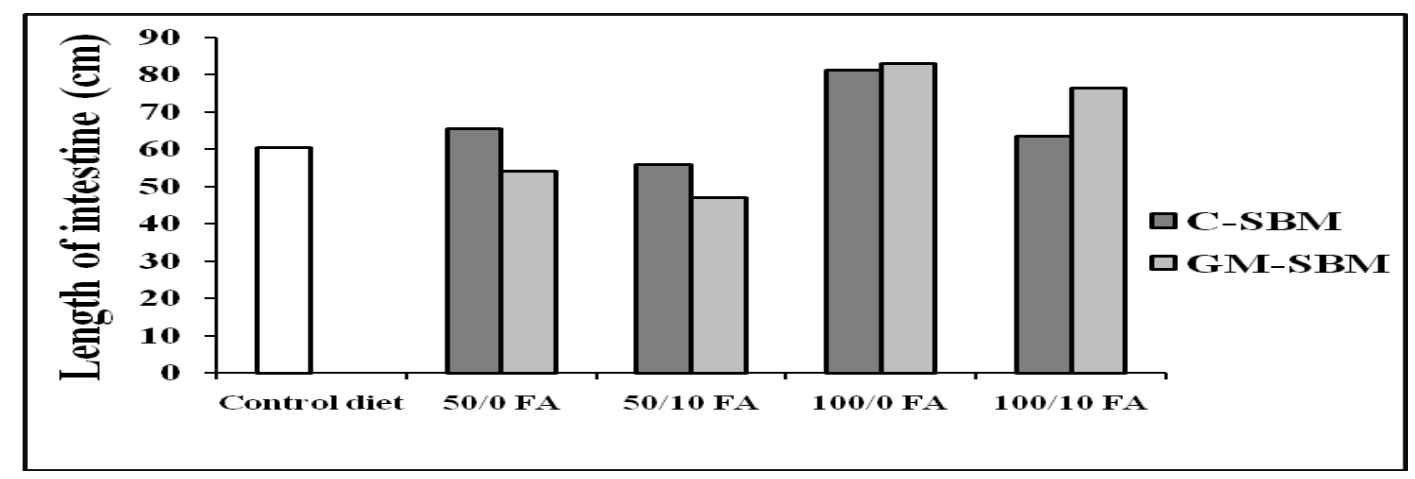

Fig. (5): Length of intestine $(\mathrm{cm})$ of Nile tilapia fed control diet and diets substituted with partial and total of dietary FM by C-SBM or GM-SBM with or without FA supplementation. Note that, length of intestine was decreased with the addition of formic acid for C-SBM and GM-SBM. Fish fed diet containing GM-SBM 100 un-supplemented with formic acid was recorded the highest value of length of intestine in comparison with control diet.

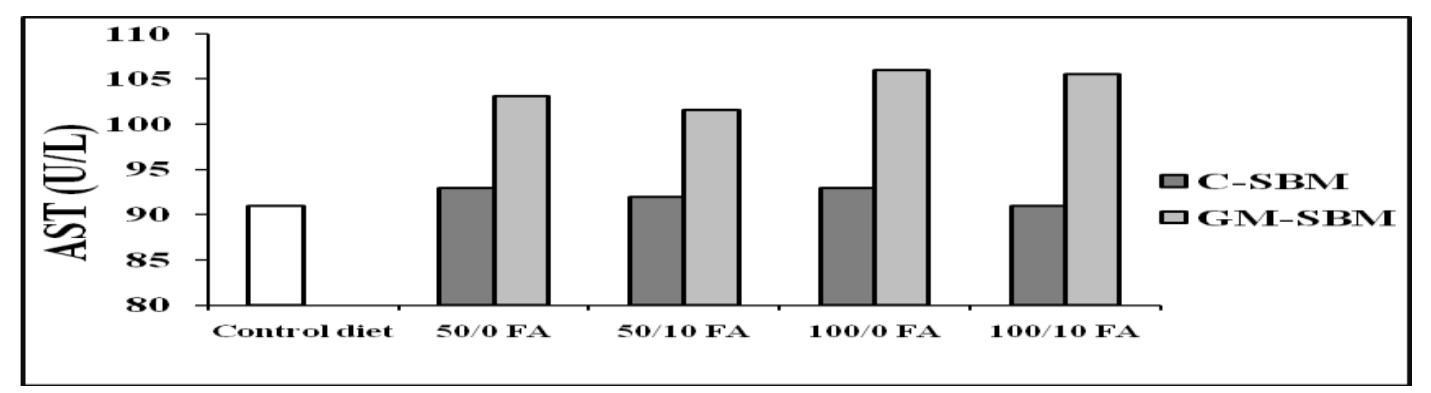

Fig. (6): AST (U/L) of Nile tilapia fed control diet and diets substituted with partial and total of dietary FM by CSBM or GM-SBM with or without FA supplementation. Note that, the lowest average of AST was observed with fish fed control diet and C-SBM. Fish fed diets supplemented with FA showed the lowest average of AST than fish fed un-supplemented diets. Fish fed diets with GM-SBM showed the highest values for AST which indicates disturbances in liver function. 


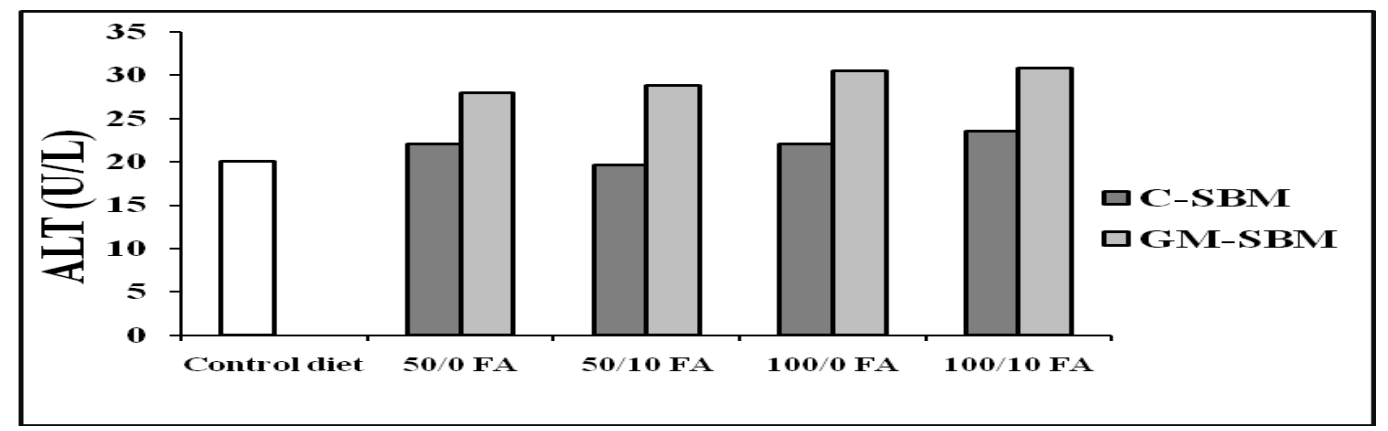

Fig. (7): ALT (U/L) of Nile tilapia fed control diet and diets substituted with partial and totally of dietary FM by C-SBM or GM-SBM with or without FA supplementation. Note that, the lowest average of ALT was observed with fish fed control diet and C-SBM. Fish fed $\mathrm{C}_{-} \mathrm{SBM}_{50}$ diet supplemented with FA showed the lowest average of ALT than fish fed un-supplemented diet. Fish fed diets with GM-SBM showed the highest values for ALT which indicates disturbances in liver function.

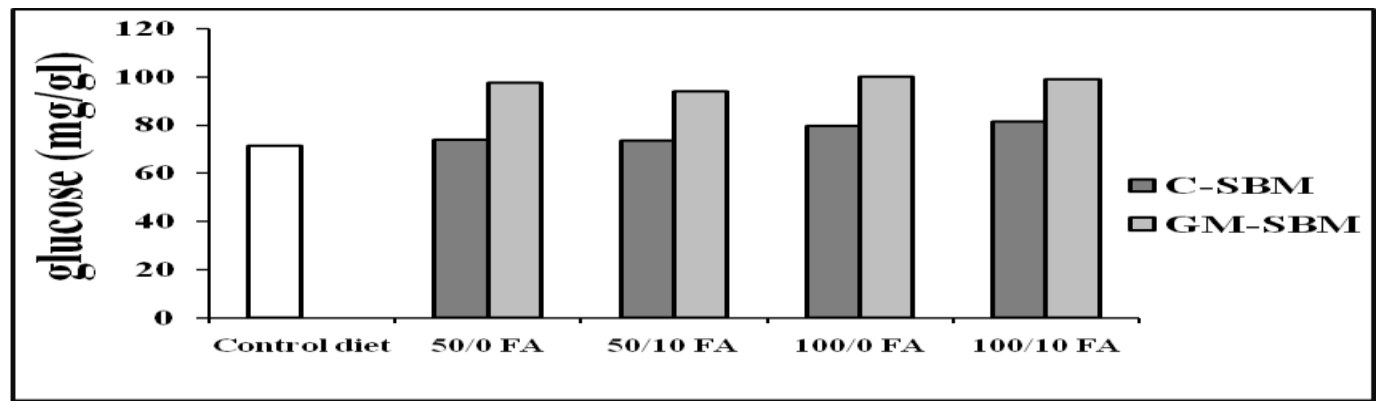

Fig. (8): Glucose (mg/gl) of Nile tilapia fed control diet and diets substituted with partial and total of dietary FM by C-SBM or GM-SBM with or without FA supplementation. Note that, the lowest average of glucose was observed with fish fed control diet and C-SBM. Glucose was decreased with formic acid supplementation for diet containing GM-SBM. The highest value of glucose was detected for diet containing un-supplemented GM-SBM 100 .

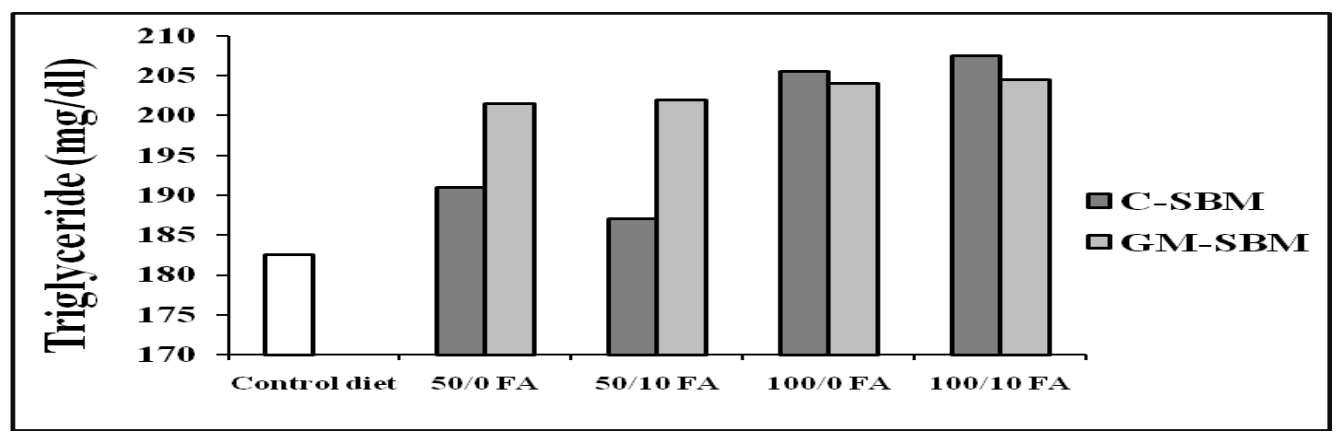

Fig. (9): Triglyceride (mg/dl) of Nile tilapia fed control diet and diets substituted with partial and totally of dietary FM by C-SBM or GM-SBM with or without FA supplementation. Note that, the lowest recorded triglyceride level was observed with fish fed the control diet followed by fish fed on $\mathrm{C}_{10}-\mathrm{SBM}_{50}$. Fish fed on $\mathrm{C}-\mathrm{SBM}_{50}$ supplemented with FA recorded less averages of triglyceride than those fed on C$\mathrm{SBM}_{50}$ un-supplemented with FA. 


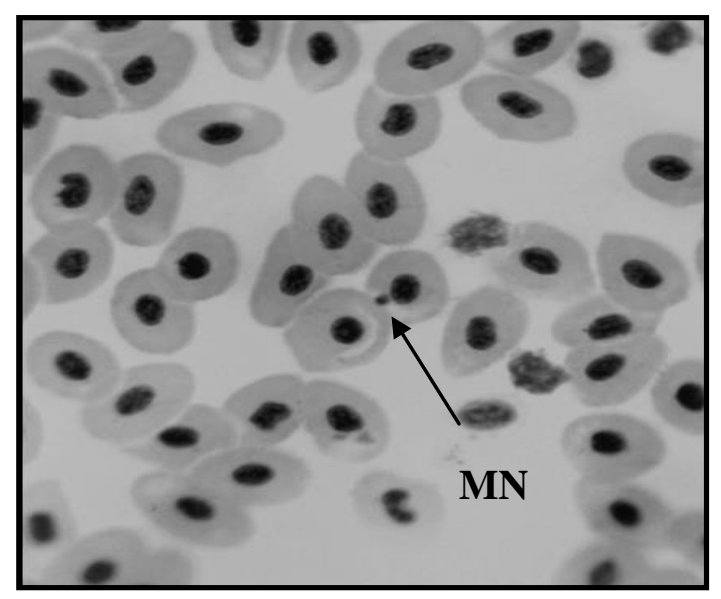

Fig. (10): Cell containing micronucleus (MN). The MN is a small rounded body - darkly stained as nucleus. It is separated from the nucleus of the cell.
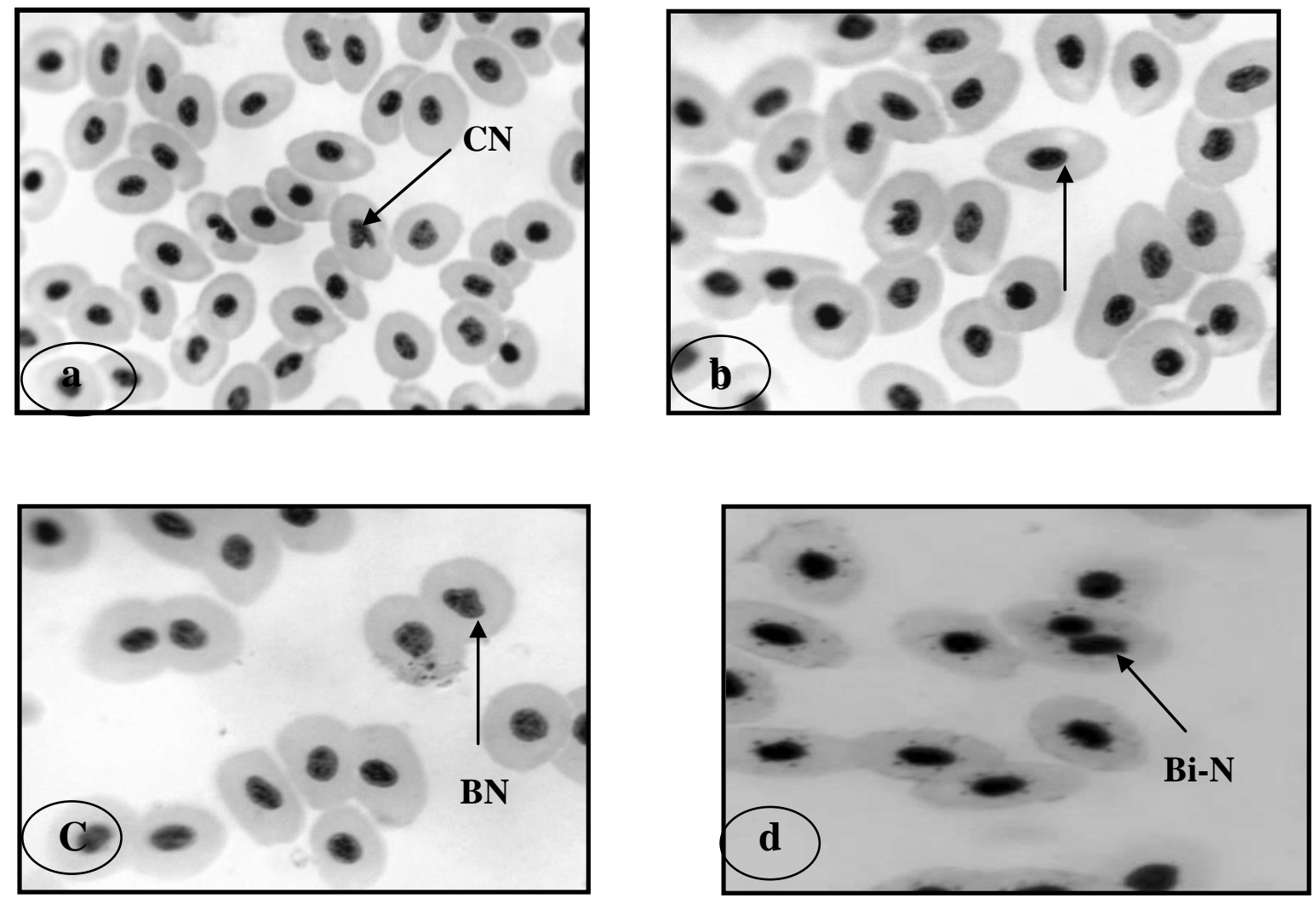

Fig. (11): Nuclear abnormalities: a) Curved nuclei $(\mathrm{CN})$ presented a relatively small evagintion of the nuclear membrane which contain euchromatin. b) Elongated nuclei (EN). c) Budding nuclei (BN): nucleus appeared as have bud on their surface. d) Bionuclated nuclei (Bi-N) characterized by cell with 2 nuclei. The two nuclei should be approximately equal size, staining pattern. 


\section{Discussion}

Aquaculture feeds typically represent up to $50 \%$ of variable operating production costs, depending on the intensity of the operation (Muzinic et al., 2006). Nowadays, a priority area of research in aquaculture nutrition is expected to reduce and possible eliminate the use of fishmeal (FM) (Craig, 2004; Goda et al., 2007).

The present study indicated that the substitution of dietary commercial FM at dietary level of $50 \%$ and $100 \%$ with either conventional soybean (C-SBM) or genetically modified soybean meal (GM-SBM) without formic acid supplementation decreased the growth performance compared to control diet. Soybean meal is considered as the best plant protein for meeting the essential amino acid requirements of tilapia and other fish species commercially grown (El-Sayed, 1999).

In our study, dietary formic acid supplementation elevated growth and feed utilization of Nile tilapia. That may be explained by some reasons. Firstly, formic acid optimized dietary protein which can decrease the amount of feed necessary for fish growth. Secondly, reduces the dietary $\mathrm{pH}$ as well as the $\mathrm{pH}$ in the intestinal lumen, which is likely to enhance the phytase activity (Erdman, 1979). Thirdly, dietary acidification may reduce the rate of gastric emptying (Mayer, 1994), which may also favor the action of phytase. Fourthly, enhancing the population of beneficial microorganism in the elementary tract and inhibiting the potential pathogens. Thus, it improved intestinal microbial balance, enzyme activity, improving feed digestibility and nutrient absorption (Sarker et al., 2005; Hassaan et al., 2014).

The present results detected slight reductions in growth of $\boldsymbol{O}$. niloticus when fed GMSBM compared to C-SBM as previously showed by Hemre et al. (2007) in Atlantic salmon, Salmo salar. However, previous studies indicated that there is no significant difference for the growth performance and feed utilization between GM-SBM and C-SBM feeding to $\boldsymbol{O}$. niloticus (Suharman et al., 2009).

Our experiment indicated that spleen size increased as the plant protein increased for CSBM and GM-SBM. According to Hemre et al. (2005) some of the plant ingredients like SBM might cause early release of immature erythrocytes which may be responsible for increased spleen size.

In the present study, the length of intestine decreased with the addition of formic acid for fish fed C-SBM and GM-SBM diets. Supplementation with organic acids reduces intestinal $\mathrm{pH}$, binds various cations along the intestine and may act as a chelating agent (Ravindran and Kornegay, 1993). As regardless of FA supplementation, the length of intestine increased as plant protein increased which concur with Kumer et al. (2010). Increasing the length of the fish intestine may be attributed to the increasing the activity of digestion, to achieve a digestive 
balance and to fit the increase in dietary plant protein content of SBM. Buddington et al. (1997) reported that omnivorous fish require longer time to digest plant protein than the animal proteinbased diets.

Hematology is an important for the assessment of suitability of diets and feed mixtures, fish physiological conditions, toxic tool effect of substances, as well as the diagnosis of disease (Svobodová et al., 1991). In the present study, $\mathrm{Hb}$ and Hct values increased in fish fed diets supplemented with FA. This result was previously reported by Baruah $\boldsymbol{e t ~ a l . ~ ( 2 0 0 7 ) ~ i n ~ R o h u ~ ( L . ~}$ Rohita) and Khajepour et al. (2011) in Juvenile Beluga (Huso huso). The increase in $\mathrm{Hb}$ and Hct may be attributed to the effect of formic acid leading to the maximum liberation and utilization of $\mathrm{Ca}, \mathrm{P}, \mathrm{Fe}$ and $\mathrm{Cu}$ from the phytic acid complex. The lowest levels of triglycerides were recorded in fish fed control diet and $\mathrm{C}_{10}-\mathrm{SBM}_{50}$. However, Saponins in different soybean strains were suggested to be responsible for elevated triglycerides (Sagstad et al., 2008).

In the present study, the lowest values of ALT and AST were observed for fish fed control diet and C-SBM diet which indicate the healthy physiological state of liver. Generally, ALT and AST levels were higher in blood samples of fish fed GM-SBM than C-SBM. ALT and AST enzymes have the function of transferring amino group from alpha-amino acids to alphaketo acids. Large amounts of ALT and AST in serum level indicated liver cell damage (Soltan $\boldsymbol{e t}$ al., 2008).

The current experiment indicated that the supplementation of FA in fish fed C-SBM 50 and GM-SBM 100 had significantly $(\mathrm{P}<0.05)$ increased the level of serum protein. This finding concurs with Baruah et al. (2007). Wiegertjes et al. (1996) observed that the increase in serum protein level is an indicator of innate immunity, which is considered as an important defense weapon mechanism of fish (Magnado' ttir, 2006).

The micronucleated erythrocytes were very low in numbers and did not show a clear differences between fish fed different diets. The frequencies ranged from 0 to 2 micronuclated erythrocyte / 2000 erythrocytes. In addition, the nuclear abnormalities in erythrocytes appeared in the form of curved, elongated, bionuclated and budding nucleus (Carrasco et al., 1990). However, their numbers were low and ranged from 2 to 4 cells / 2000 erythrocytes. There was no correlation between their numbers and fish feed different diets.

In the present study, whole body ash content increased with FA supplementation in fish fed either C-SBM or GM-SBM diets. As proved previously by Hassaan et al. (2013) this may be due to addition of organic acid which enhances minerals absorption through the intestine for Nile tilapia. Similar results were observed in the previous findings of Sarker et al. (2012) in juvenile yellowtail, Seriola quinqueradiata and Khajepour and Hosseini (2012) in Beluga (Huso huso). Our results induced that lipid content increased in fish fed either GM-SBM or C-SBM 
diets with FA supplementation. This finding was concurred with Khajepour and Hosseini (2012). The present study showed that, FA supplementation increased protein content for different experiment diets.

Overall view showed that the inconsistency in the outcome of the present investigation as well as previous studies which may be attributed to differences in feed ingredients, nutritional quality of plant ingredients, water quality, fish species and size and culture or experimental conditions (Hassaan et al., 2013).

\section{Conclusion}

Formic acid supplementation enhanced the growth performance, feed utilization and biological abdominal parameters in fish fed either $\mathrm{C}_{-} \mathrm{SBM}_{50}$ or $\mathrm{GM}-\mathrm{SBM}_{50}$. As regarding the genetically modified soybean it needs more investigation to evaluate their effect on feeding trial on fish and credit it as a safe reproducible source in fish.

\section{Acknowledgment}

The authors have no financial conflict of interest.

\section{References}

Abdel-Warith, A.A; Younis, E. M. and Abdualla, N. A. , Influence of dietary inclusion of fullfat soybean meal and amino acids supplementation on growth and digestive enzymes activity of Nile tilapia, Oreochromis niloisticus. Turkish J. Fish. Aquac. Sci., 13: 69-77 (2013).

Adewolu, MA and Adoti, AJ., Effect of mixed feeding schedules with varying dietary crude protein levels on the growth and feed utilization of Clarias gariepinus (Burchell, 1822) fingerlings. J. Fish. Aquac. Sci., 5940: 304-310 (2010).

AOAC , In: Cunni, P.A. (Ed.), Official Methods of Analysis of the Association Official Analytical Chemists, vol. 1, 16th ed. AOAC International, Arlington, USA, p. 1298 (1995).

Baruah, K.; Sahu, NP.; Pal, AK.; Jain, KK.; Debnath, D. and Mukherjee, SC. , Dietary microbial phytase and citric acid synergistically enhances nutrient digestibility and growth performance of Labeo rohita (Hamilton) juveniles at sub-optimal protein level. Aquacul. Res., 38: 109-120 (2007).

Bergmeyer, H. U., Methods of enzymatic analysis. Deerfield Beach: VCH (1986).

Boyd, C. E. , Water quality in ponds for aquaculture. Alabama Agriculture Experiment Station, Auburn University, Al., p. 482 (1990).

Brett, J.R. , Energy expenditure of Sockeye salmon Oncorhynchus nerka, during sustained performance. J. Fish. Res. Board Can., 30: 1799-1809 (1973). 
Brown, P.B.; Wilson, K.; Jonker, Y. and Nickson, T.E. , Glyphosate tolerant canola meal is equivalent to the parental line in diets fed to rainbow trout. J. Agric. Food Chem., 51: 42684272 (2003).

Buddington, R.K.; Krogdahl, Å. and Bakke-McKellep, A.M. , The intestines of carnivorous fish: structure and functions and the relations with diet. Acta. Physiol. Scand., 161: 67-80 (1997).

Carrasco, KR.; Tilbury, KL. and Mayers, MS. , Assessment of the piscine micronuclei test as an in situ biological indicator of chemical contaminants effects. Can. J. Fish. Aquac. Sci., 47: 2123-2136 (1990).

Coles, E.H., Plasma proteins. In: Veterinary clinical pathology, $2^{\text {nd }}$ edition. W.B. Saunders Co., Philadelphia, Pennsylvania, USA, pp. 558-560 (1974).

Craig, S. R. , Organic agricultural feed. Aqua feeds: Formulation Beyond, (1): 11-13 (2004).

Da Silva, B.C.; Vieira, F.; Mouriño, J.L.; Ferreira, G.S. and Seiffert, W.Q. , Salts of organic acids selection by multiple characteristics for marine shrimp nutrition. Aquaculture, 384387: 104-110 (2013).

De Flora, S.; Vigano, L.; Agostini, F.D.; Camoirano, A.; Bagnusio, M.; Bennieeli, C., et al. , Multiple genotoxicity biomarkers fish exposed in situ to polluted river water. Mutat. Res., 319:167-77 (1993).

Duncan, N. B. , Multiple ranges and multiple F-tests. Biometrics, 11: 1-24 (1955).

El-Sayed, A.F.M. , Alternative dietary protein sources for farmed tilapia, Oreochromis spp. Aquaculture, 179(1-4):149-168 (1999).

Erdman, Jr., J.W. , Oilseed phytates: nutritional implications. J. Am. Oil Chem. Soc., 56: 736741 (1979).

Goda, A.S.A.S.; Wafa, M.E.; El-Haroun, E.R. and Chowdhury, M. A. K. , Growth performance and feed utilization of Nile tilapia Oreochromis niloticus (Linnaeus, 1758) and tilapia galilae Sarothrodon galilaeus (Linnaeus, 1758) fingerlings fed plant protein-based diets. Aquacult. Res., 38: 827-837 (2007).

Hardy, R.W. , Utilization of plant proteins in fish diets: effects of global demand and supplies of fishmeal. Aquacult. Res., 41: 770-776 (2010).

Hassaan, M.S.; Soltan, M.A.; Agouz, H.M. and Badr, A.M. , Influences of calcium/phosphorus ratio on supplemental microbial phytase efficiency for Nile tilapia (Oreochromis niloticus). Egypt. J. Aquac. Res., 39: 205-213 (2013).

Hassaan, M.S.; Wafa, M.A.; Soltan, M. A.; Goda, A.S. and Mogheth, N.M.A. , Effect of dietary organic salts on growth, nutrient digestibility, mineral absorption and some biochemical indices of Nile Tilapia; Oreochromis niloticus L. fingerlings. World Appl. Sci. J., 29 (1): 47-55 (2014).

Hemre, G.I.; Sanden, M.; Bakke-Mckellep, A.M.; Sagstad, A. and Krogdahl, A. , Growth, feed utilization and health of Atlantic salmon Salmo salar L. fed genetically modified compared to non-modified commercial hybrid soybeans. Aquac. Nutr., 11: 157-167 (2005). 
Hemre, G.I. ; Sagstad, A. ; Bakke-Mckellep, A.M. ; Danieli, A. ; Acierno, R. ; Maffia, M. ; Frøystad, M. ; Krogdahl, A. and Sanden, M. , Nutritional, physiological, and histological responses in Atlantic salmon, Salmo salar L. fed diets with genetically modified maize. Aquac. Nutr., 13: 186-199 (2007).

Henry, R.J. , Colorimetric determination of total protein. In: Clinical Chemistry. New York: Harper and Row Publ (1964).

Jouany, J.-P. and Morgavi, D. P. , Use of natural products as alternative to antibiotic feed additives in ruminant production. Anim., (1:10): 1443-1446 (2007).

Kaushik, S.J. and Hemre, G.-I. , Plant proteins as alternative sources for fish feed and farmed fish quality. In improving farmed fish quality and safety (Lie, Ø. ed.), pp. chapter 12, pp. 300-319. Wood head Publishing Limited, CRC Press, Cambridge, England (2008).

Khajepour, F.; Hosseini, S.A. and MaHoseini, S. , Study on some hematological and biochemical parameters of juvenile Beluga (Huso huso) fed citric acid supplemented diet. Global Veterinaria, 7: 361-364 (2011).

Khajepour, F. and Hosseini, S.A. , Citric acid improves growth performance and phosphorus digestibility in Beluga (Huso huso) fed diets where soybean meal partly replaced fish meal. Anim. Feed Sci. Technol., 171: 68-73 (2012).

Kumar, V.; Makker, H.P.S. and Becker, K. , Detoxified Jatropha curcas kernel meal as a dietary protein source: growth performance, nutrient utilization and digestive enzyme in common carp fingerlings. Aquacult. Nutrit., Dio: 10.111/j. 1365-2095 (2010).

Liebert, F.; Mohamed, K. and Lückstädt, C. , Effects of diformates on growth and feed utilization of all male Nile Tilapia fingerlings (Oreochromis niloticus) reared in tank culture. XIV International Symposium on Fish Nutrition and Feeding, Qingdao, China, Book of Abstracts pp: 190 (2010).

Lim, C.; Lückstädt C. and Klesius, P. H. , Review: use of organic acids and salts in fish diets. Global Aquacult. Advocates, 5: 45-46 (2010).

Magnado' ttir, B. , Innate immunity of fish (overview). Fish and Shellfish Immunol., 20: 137151 (2006).

Mayer, E.A. , The physiology of gastric storage and emptying. In: Physiology of the gastrointestinal tract. Volume 1, Johnson, L.R. (Ed.). New York: Raven Press (1994).

Meyer, R. , Development and application of DNA analytical methods for the detection of GMOs in food. Food Basal, 10: 391-399 (1999).

Morken, T.; Kraugerud, O.F. ; Barrows, F.T. ; Sørensen, M. ; Storebakken, T. and Øverland, M. , Sodium diformate and extrusion temperature affects nutrient digestibility and physical quality of diets with fish meal and barley protein concentrate for rainbow trout (Oncorhynchus mykiss). Aquaculture, 317(1-4): 138-145 (2011).

Muzinic, L.A.; Thompson, K.R.; Metts, L.S.; Dasgupta, S. and Webster, C.D. , Use of turkey meal as partial and total replacement of fishmeal in practical diets for sunshine bass (Morone chrysops $\times$ Morone saxatiilis) grown in tanks. Aquacult. Nutr., (12) : 71-81 (2006). 
Padgette, S.R.; Kolacz, K.H.; Delannay, X.; Re, D.B.; LaVallee, B.J.; Tinius, C.N.; Rhodes,W.K.; Otero,Y.I.; Barry,G.F.; Eichholtz, D.A.; Peschke,V.M.; Nida, D.L.; Taylor, N.B. and Kishore, G.M. , Development, identification, and characterization of a glyphosate-tolerant soybean line. Crop Sci., 35: 1451-1461 (1995).

Ravindran, V. and Kornegay, ET. , Acidification of weaner pig diets: a review. J. Sci. Food. Agric., 62: 313-22 (1993).

Reitman, A. and Frankel, S. , Determination of aspartat glutamic aminotransferase and alanin aminotansferase. Am. J. Clin. Pathol., 28: 56-59 (1957).

Sagstad, A.; Sanden, M. ; Krogdahl, Á.; Bakkle-Mckellep, A.M.; Frøystad, M. and Hemre, G.I. , Organs development, gene expression and health of Atlantic salmon (Salmo salar L.) fed genetically modified soybeans compared to the near-isogenic non-modified parental line. Aquac. Nutr., 14: 556-572 (2008).

Sanden, M.; Berntssen, M.H.G.; Krogdahl, Å.; Hemre, G.I. and Bakke-McKellep, A.M. , An examination of the intestinal tract of Atlantic salmon, Salmo salar L., parr fed different varieties of soy and maize. J. Fish Dis., 28: 317-330 (2005).

Sarker, MSA; Satoh, S and Kiron, V. , Supplementation of citric acid and amino acid-chelated trace element to develop environment friendly feed for red sea bream, Pagrus major. Aquaculture, 248: 3-11 (2005).

Sarker, M.S.A.; Satoh,S.; Kamata,K.; Haga, Y. and Yamamoto, Y. , Supplementation effect(s) of organic acids and/or lipid to plant protein-based diets on juvenile yellowtail, Seriola quinqueradiata Temminck et Schlegel 1845, growth and, nitrogen and phosphorus excretion. Aquacult. Res., 43: 538-545 (2012).

Soltan, M.A. ; Hanafy, M.A. and Wafa, M.I.A. , Effect of replacing fishmeal by a mixture of different plant protein sources in Nile Tilapia (Oreochromis niloticus L.) diets. Global Veterinaria, 2 (4): 157-164 (2008).

Statistical Analysis system , SAS/STAT user Guide Release 6.03 Edition. SAS Institute Inc. Cary, North Carolina, USA (1993).

Suharman, I.; Satoh, S.; Haga, Y.; Takeuchi, T.; Endo, M.; Hirono, I. and Aoki, T. , Utilization of genetically modified soybean meal in Nile tilapia Oreochromis niloticus diets. Fish Sci., 75: 967-973 (2009).

Svobodová, Z.; Fravda, D. and Palakova, J. , Unified methods of hematological examination of fish. Research Institute of Fish Culture and Hydrobiol., VURH Vodnany, Edice Metodik, Czechoslovakia (1991).

Vielma, J. and Lall, S. P. , Dietary formic acid enhances apparent digestibility of minerals in rainbow trout, Oncohynchus mykiss (Walbaum). Aquacult. Nutri., 3, issue 4: 265-268 (1997).

Wiegertjes, G.F.; Stet, R.J.M. ; Parmentier, H.K. and Van Muiswinkel., W.B. , Immunogenetics of disease resistance in fish: a comparable approach. Dev. Comp. Immunol., 20: 365-381 (1996). 
Wotton, I.D. and Freeman, H. , Microanalysis in Medical Biochemistry. Churchill, New York. USA. Wiegertjes, G.F., R.J.M. Stet, H.K. Parmentier and W.B. Van Muiswinkel, 1996. Immunogenetics of disease resistance in fish; a comparable approach Dev. Comp. Immunol., 20: 365-381 (1982).

Yigit, N. O. and Olmez, M. , Canola meal as an alternative protein source in diets for fry of Tilapia (Oreochromis niloticus). The Israeli J. Aquacult. - Bamidgeh, 61(1): 35-41 (2009).

Yigit, M; Ergün, S; Türker, A; Harmantepe, B. and Erteken, A. , Evaluation of soybean meal as a protein source and its effect on black Sea turbot (Psetta maeotica) juveniles. J. Mar. Sci. Technol., 18(5): 682- 688 (2010).

Zar, J. H. , Biostatistical Analysis. Prentice-Hall, Englewood Cliff, NJ, USA (1984).

Zhou, Z.; Liu, Y.; He, S; Shi, P.; Gao, X.; Yao, B. and Ringø, E. , Effects of dietary potassium diformate $(\mathrm{KDF})$ on growth performance, feed conversion and intestinal bacterial community of hybrid tilapia (Oreochromis niloticus $\times$ O. aureus). Aquaculture, 291 (1-2): 89-94 (2009).

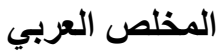

$$
\begin{aligned}
& \text { الأحلال الجزئي والكلي لمسحوق السمك بفول الصويا التقليدي أو المعدل ور اثيا مضافا أو غير مضافا إليه حمض الفض الفيات } \\
& \text { الفورمك لاصبباعيات البلطي النيلي. }
\end{aligned}
$$

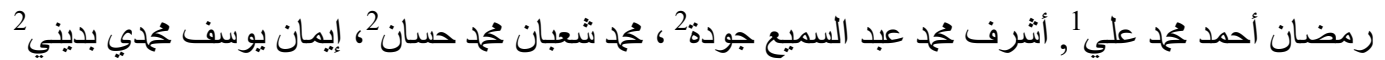

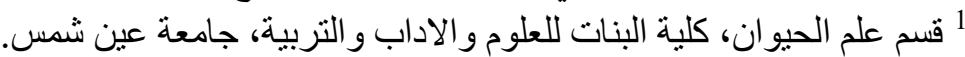

$$
\begin{aligned}
& 2 \text { معمل تغذية الأسماك، المعهد القومي لعلوم البحار و المصايد، محطة بحوث الاسماك، القناطر الخيرية. }
\end{aligned}
$$

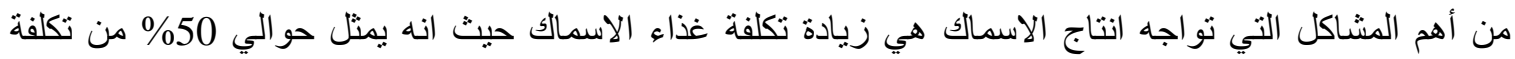

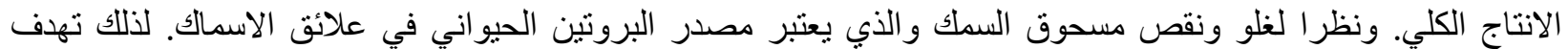

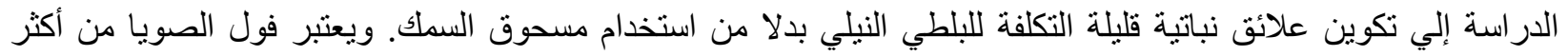

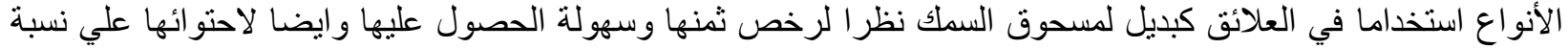

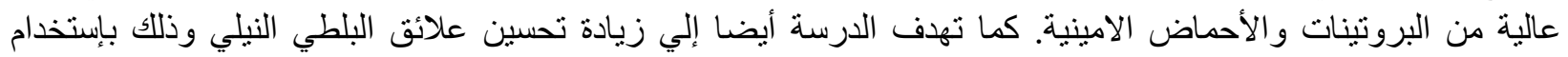
اضافات مثل الأحماض العضوية.

في هذا البحث تم إجر اء تجربة تغذية وذلك بأستبدال مسحوق السمك جزيئا أو كليا بفول الصويا التقليدي أو المعدل

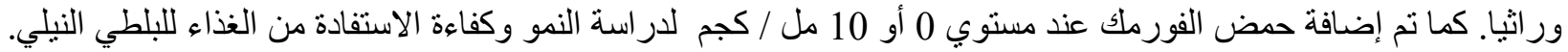

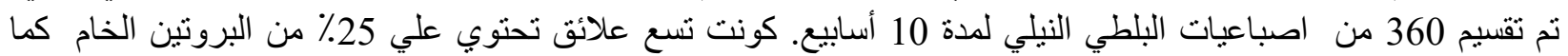
تحتوي ايضا علي طاقة 19,28 ميجاجول / كيلو جر ام من الطاقة الإجمالية.

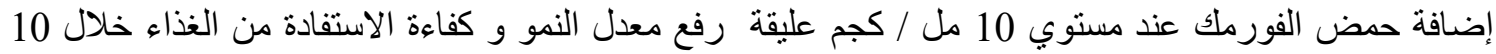

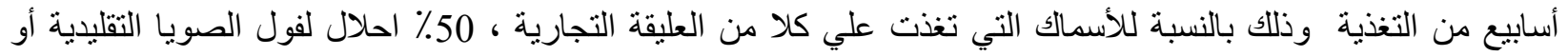

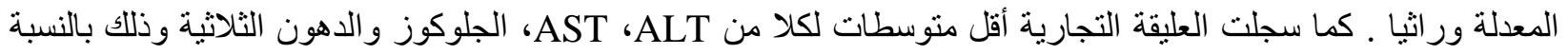

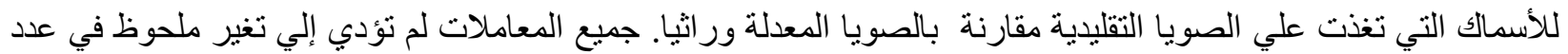

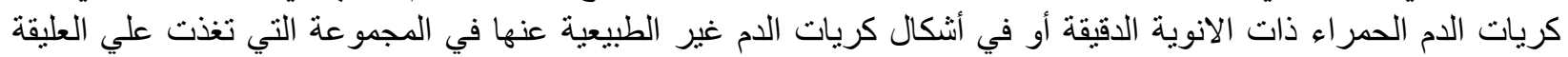
التجارية. 


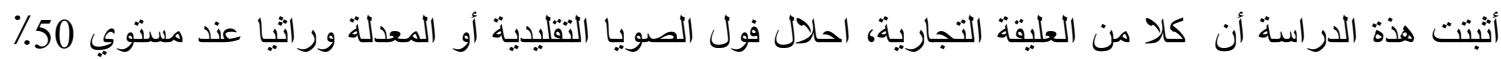

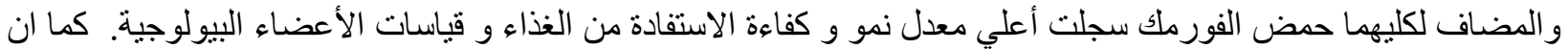

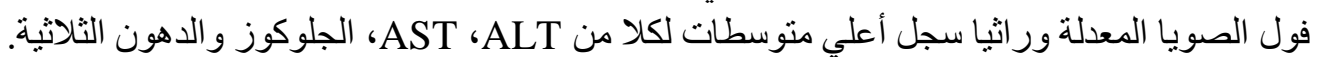

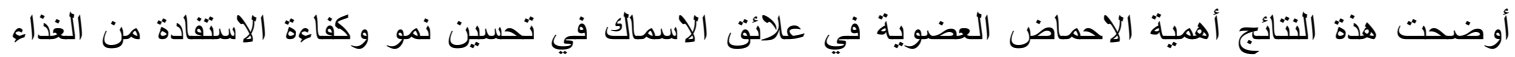

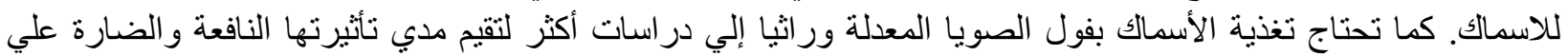

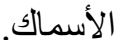

